\title{
Visualizing the RNP content of single bunyavirus virions reveals more efficient genome packaging in the arthropod host
}

\section{Erick Bermúdez-Méndez}

Wageningen Bioveterinary Research https://orcid.org/0000-0001-9241-7898

\section{Eugene Katrukha}

Faculty of Science, Utrecht University https://orcid.org/0000-0002-9971-3603

Cindy Spruit

Utrecht University

Jeroen Kortekaas

Wageningen Bioveterinary Research

Paul Wichgers Schreur ( $\nabla$ paul.wichgersschreur@wur.nl)

Wageningen Bioveterinary Research

\section{Article}

Keywords: Bunyaviruses, genome packaging, arthropod

Posted Date: September 4th, 2020

DOl: https://doi.org/10.21203/rs.3.rs-49677/v1

License: (c) (1) This work is licensed under a Creative Commons Attribution 4.0 International License. Read Full License

Version of Record: A version of this preprint was published at Communications Biology on March 22nd, 2021. See the published version at https://doi.org/10.1038/s42003-021-01821-y. 
1 Visualizing the RNP content of single bunyavirus virions reveals more

2 efficient genome packaging in the arthropod host

3

4

5

6

7

8

9

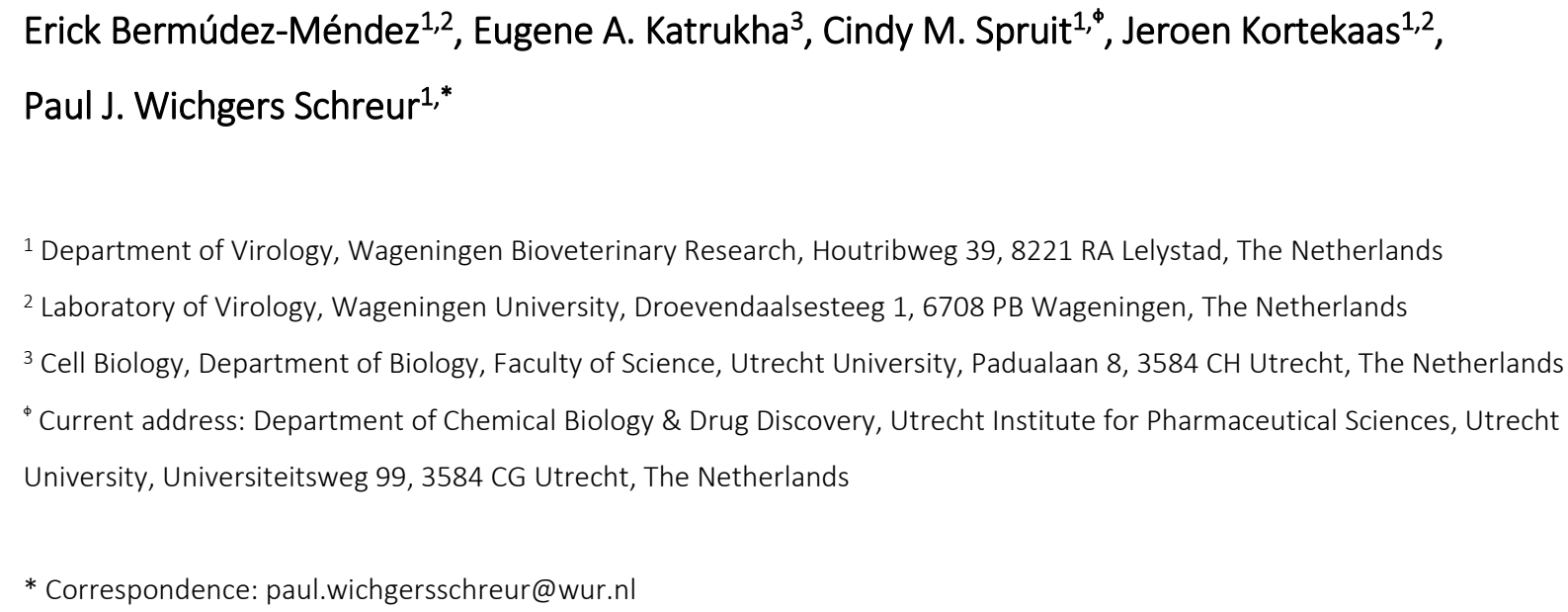

\begin{abstract}
Bunyaviruses have a genome that is divided over multiple segments. Genome segmentation complicates the generation of progeny virus, since each newly formed virus particle should preferably contain a full set of genome segments in order to disseminate efficiently within and between hosts. Here, we combine immunofluorescence and fluorescence in situ hybridization techniques to simultaneously visualize bunyavirus progeny virions and their genomic content at single-molecule resolution in the context of singly infected cells. Using Rift Valley fever virus and Schmallenberg virus as prototype tri-segmented bunyaviruses, we show that bunyavirus genome packaging is influenced by the intracellular viral genome content of individual cells, which results in greatly variable packaging efficiencies within a cell population. We further show that bunyavirus genome packaging is more efficient in insect cells compared to mammalian cells and provide new insights on the possibility that incomplete particles may contribute to bunyavirus spread as well.
\end{abstract}




\section{Introduction}

Viruses from the genera Phlebovirus (family Phenuiviridae) and Orthobunyavirus (family Peribunyaviridae), belonging to the order Bunyavirales, are globally distributed and transmitted between vertebrate hosts by arthropods such as mosquitoes, sandflies, ticks and midges ${ }^{1-4}$. Several members of these genera cause severe disease in livestock and humans, threatening animal and public health and economies ${ }^{5,6}$. Yet, several fundamental aspects of the viral life cycles remain poorly comprehended.

Phleboviruses and orthobunyaviruses have a tri-segmented genome of single-stranded RNA of negative-sense polarity. The small (S), medium (M) and large (L) segments, named according to their size, are encapsidated by multiple nucleocapsid $(\mathrm{N})$ proteins to form viral ribonucleoprotein (vRNP) complexes that associate with the RNA-dependent RNA polymerase (RdRp or L protein). The $\mathrm{N}$ protein is encoded by the S segment, which also encodes a non-structural protein (NSs) in antigenomic-sense orientation in phleboviruses and in genomic-sense orientation in orthobunyaviruses. The RdRp is encoded by the L segment, whereas the M segment encodes a polyprotein precursor that is cleaved into a non-structural protein (NSm) and two glycoproteins (Gn and Gc) that protrude from the envelope of mature particles and facilitate entry into host cells ${ }^{6-8}$. Virions are enveloped, spherical particles of approximately $80-120 \mathrm{~nm}$ in diameter ${ }^{9-11}$.

From a gene expression perspective, genome segmentation could theoretically facilitate control of viral gene transcription and translation without requiring various cis-acting elements as viruses with non-segmented genomes require. Moreover, genome segmentation is generally considered as an evolutionary advantage because it allows genetic reassortment events, which can potentially result in increased viral fitness and transmissibility ${ }^{12}$. However, partitioning of the genome complicates the genome packaging process of segmented viruses, since packaging of at least one copy of each segment into a particle is thought to be essential to generate infectious progeny. Considering this, it could be expected that packaging of segmented viral genomes is a highly selective process. The existence of a selective packaging mechanism has already been demonstrated for segmented RNA viruses of other families such as influenza virus and rotavirus ${ }^{13-15}$. Reverse genetics and electron microscopy studies on influenza virus showed that the eight genome segments use packaging signals to assemble into a supramolecular complex with a ' $7+1$ ' configuration ${ }^{16-19}$. Fluorescence spectroscopy combined with pulsed interleaved excitation revealed that rotavirus genome segments form protein-mediated sequence-specific interactions ${ }^{20}$. In both cases, RNA-RNA interactions play an important role in the packaging of the complete genome inside newly formed particles.

Early reports based on mini-genome systems showed that the 5' and 3' untranslated regions (UTRs) of bunyavirus RNA segments are directly or indirectly involved in the genome packaging process ${ }^{21}$. 
Certain flexibility in the packaging process was demonstrated by the rescue of a recombinant Bunyamwera virus (BUNV, genus Orthobunyavirus) with an L segment open reading frame flanked by M-type UTRs ${ }^{22}$. Additional work with recombinant viruses revealed the flexibility in the packaging of Rift Valley fever virus (RVFV, genus Phlebovirus) genome segments, as evidenced by the creation of multiple two-segmented and four-segmented variant ${ }^{23-25}$, as well as a variant with reconfigured coding orientation of the $\mathrm{S}$ segment $^{26}$. More recently, by using single-molecule fluorescence in situ hybridization (smFISH) we showed that S, M and L vRNPs of RVFV do not co-localize in the cytoplasm during viral replication. Together with a codon shuffled M-segment variant that retained similar growth characteristics, no evidence was found for the formation of a supramolecular RVFV vRNP complex, thereby suggesting that packaging of RVFV genome segments is not a tightly regulated process ${ }^{27}$. Despite that the scarce evidence available has provided valuable insights into the genome packaging of bunyaviruses, our understanding of this process is still very limited. In particular, packaging of bunyaviruses has only been studied with a few virus species, and few studies have compared genome packaging in different hosts. Potential host differences in specific steps of the replication cycle may have important implications for virus transmission between vertebrates and invertebrates. Additionally, the kinetics and efficiency of generating infectious particles have only been examined at a cell population level and the potential biological role of incomplete particles (i.e. particles lacking one or more genome segments) in within- and between-host transmission is currently unknown.

Here, we use RVFV and Schmallenberg virus (SBV, genus Orthobunyavirus) as prototypes of different bunyavirus families to study genome packaging in mammalian and insect cells. We describe a 5-channel FISH-immunofluorescence method that allows simultaneous visualization of progeny virions and each viral genome segment at single-molecule resolution, directly showing that only a small fraction of newly formed virus particles contains a full set of genome segments. We further show at a single-cell level that the packaging efficiency is highly heterogeneous within a cell population and provide direct evidence of the occasional incorporation of antigenomic-sense segments into virus particles. Finally, we report major differences between genome packaging efficiencies in mammalian and insect cells. Thus, the results of this study are in line with our previous suggestion that genome packaging of bunyaviruses is driven by a non-selective process and highlight host cell differences in bunyavirus life cycles.

\section{Results}

\section{Viral RNA:infectivity ratios differ in mammalian and insect hosts}

To study viral replication and the generation of infectious virus progeny in mammalian and insect cells, we infected Vero E6 (monkey), C6/36 (Aedes albopictus) and KC (Culicoides sonorensis) cells with RVFV or SBV, quantified in time intracellular and extracellular viral genome segments by RT-qPCR and determined the virus titer in the supernatant by endpoint titration (Fig. 1a). For both RVFV and 
SBV, the absolute genome segment copy numbers of all three segments were higher in lysates and supernatants of mammalian cells (Vero E6) compared to insect cells (C6/36 and $\mathrm{KC}$ ) in the logarithmic viral growth phase (Fig. 1b-e). Remarkably, the higher genome copies in supernatants of Vero E6 cells did not always correspond proportionally with higher virus titers. For example, RVFV genome copies obtained at $48 \mathrm{~h}$ post-infection in Vero E6 cells were more than 10 times higher than in C6/36 cells, whereas the virus titers in both host cell lines were equal (Fig. 1f). Another dissonance was observed with SBV at 24 h post-infection, where similar genome copies in supernatants of Vero E6 and KC cells resulted in a titer of infectious virus more than 10 times higher in $\mathrm{KC}$ cells (Fig. 1g). After relating viral RNA copy numbers with virus titers of the supernatants in time, here referred to as vRNA:infectivity ratios, it became clear that for the generation of RVFV and SBV infectious units, fewer genome equivalents are needed in insect cells (Fig. 1h, i), suggesting that bunyavirus genome packaging efficiencies differ between hosts. In addition, genome copies in plasma samples of lambs experimentally infected with RVFV within the scope of another study ${ }^{28}$ were determined (Fig. 1j-1). Briefly, lambs were inoculated via intravenous route with RVFV, followed by daily collection of plasma samples. In these plasma samples, vRNA:infectivity ratios increased over time, with the lowest ratio observed at 2 days post-infection (Fig. 1m), coinciding with peak viremia and the onset of symptoms ${ }^{28}$, demonstrating that genome packaging efficiencies within a host may differ in time.

\section{Visualization of newly formed progeny virions at single-particle resolution}

To investigate the release kinetics of progeny virions from infected cells, we developed an immunofluorescence assay using antibodies targeting the surface glycoproteins of RVFV (Gn) and SBV (Gc). We infected Vero E6 cells, fixed the cells at defined time points and tracked the appearance of virus particles over time (Fig. 2a). For both RVFV and SBV, detection of the glycoproteins became evident around $5 \pm 1 \mathrm{~h}$ post-infection. In the case of RVFV, the Gn glycoprotein signal started to accumulate in a perinuclear region (Fig. 2b, first panel), consistent with the Golgi apparatus being the site of virion assembly ${ }^{29}$. No accumulation of Gc in perinuclear regions was noticed in SBV infected cells (Fig. 2c). Interestingly, around $7 \pm 1$ h post-infection with RVFV, localized clusters of symmetric spots, most likely portraying groups of virus particles trafficking simultaneously from the assembly site to the extracellular space in vesicles, were detected (Fig. 2b, second panel). As the infection progressed, a higher number of virus particles (hundreds to a few thousands) both inside and outside infected cells were detected (Fig. 2b-e and Supplementary Movies 1,2). Of note, we commonly found infected cells at different stages of infection despite being fixed at the same time point, probably representing the intrinsic cell-to-cell variability within a cell population regarding virus entry and viral gene expression. A plot of fluorescence intensities of individual spots shows a unimodal intensity distribution characteristic of single particles (Fig. 2f). Likewise, a histogram of the area of the spots also shows a unimodal distribution, denoting reproducible measurements of single spots within and between images (Fig. 2g). Importantly, the single-particle detection of newly formed progeny virions not only allowed 
us to investigate the kinetics of virion release, but also enabled us to determine the genomic composition of individual progeny virions.

\section{Genome composition of newly formed virus particles}

To investigate the genome content of newly formed RVFV and SBV virions in infected cells, we developed a 5-channel based combined RNA FISH-immunofluorescence method that allows simultaneous visualization of virus particles and each viral genome segment at single-molecule resolution (Fig. 3a). Virions were detected as described in Fig. 2 and specific FISH probe sets were designed to recognize the S, M and L viral RNAs (Supplementary Figs. 1, 2 and Supplementary Table 1). The method enables the concomitant assessment of viral replication by quantification of the vRNPs in the cytoplasm (Fig. 3d and Supplementary Fig. 3), as well as the determination of the genome content of newly formed virus particles through co-localization analysis between the virions and the vRNPs (Fig. 3b, c, e, f, Supplementary Figs. 3, 4 and Supplementary Movies 3, 4). Importantly, our assay facilitates linking the genomic content of the virions with the cytoplasmic vRNP content of the originative cell.

We used the assay to analyze individual RVFV and SBV infected cells fixed at $8 \mathrm{~h}$ post-infection, a stage in the infection cycle at which release of mature virions is clearly evident (Fig. 3b, c, e, f and Supplementary Figs. 3, 4) and virus genome replication has not proceeded long enough to impede the quantification of vRNPs in the cytoplasm due to an overcrowded signal detection (Fig. $3 \mathrm{~d}$ and Supplementary Fig. 3). Following analysis, most RVFV and SBV particles were found to be empty, accounting on average for approximately $55 \%$ and $35 \%$ of total virions, respectively. In addition, the fraction of particles containing one segment was between approximately $30 \%-35 \%$, and the fraction containing two segments between about $10 \%-20 \%$. The fraction of particles containing a complete genomic set was below 10\% (Fig. 4a, right panels). Remarkably, we observed great variability in packaging efficiencies within RVFV and SBV infected cell populations. Within both cell populations, a subpopulation of cells showed a striking inefficient packaging process, in some cases seemingly without generating a single infectious particle, whereas other cell subpopulations generated two or more times higher percentages of particles containing a complete genomic set than the average (Fig. 4a, right panels).

\section{Intracellular vRNP content correlates with genome packaging efficiency}

Seeking for an explanation to the high variability in genome packaging efficiency within cell populations, we looked into the vRNP content in the cytoplasm of the individual cells. Quantification of RVFV vRNPs in infected mammalian cells not only exposed a highly heterogenous cell-to-cell composition, but also an overall imbalanced content leaning towards higher abundances of the $\mathrm{S}(42 \%)$ and L (34\%) segments compared to the M segment (24\%). Quantification of SBV vRNPs in infected 
mammalian cells demonstrated that the overall vRNP content of the cytoplasm approached a theoretical balance, with abundances near the 33\% for all three genome segments. Although the cytoplasm of some SBV infected cells deviated from the average composition, the cell-to-cell heterogeneity in this population was less pronounced (Fig. 4a, left panels).

Next, we evaluated whether an imbalanced cytoplasmic content could be associated with a particular genome composition of the virions. The correlation analysis made evident that indeed, if a specific genome segment is more abundant intracellularly, it will be incorporated into a virus particle more often, and vice versa. A strong positive correlation (Pearson's coefficients of at least 0.5660 and $p<0.01$ ) was found for all three genome segments of RVFV and SBV (Fig. 4b). The association between the cytoplasmic content and the efficiency of incorporating genome segments into virions was further assessed in a more integrative manner. Based on the frequencies of all three genome segments in the cytoplasm of individual cells and the fractions of empty, incomplete and complete particles, we generated a system to score the balance of the intracellular contents as well as the efficiency of genome packaging, normalizing the scores using the extreme values present in our data set as reference (Fig. 4c). Surprisingly, our analysis revealed that a considerable number of cells with balanced intracellular genome contents exhibited an overall inefficient packaging. This indicates that, although the three different vRNPs will most likely be incorporated into particles in similar numbers if their intracellular abundance is similar, the three vRNPs are not necessarily co-packaged into the same particle. However, when we observed relatively efficient packaging, the vRNP content in the cytoplasm was balanced, implying that a balanced intracellular vRNP content is a pre-requisite for relatively efficient genome packaging. Accordingly, it also became clear that an imbalanced cytoplasmic vRNP content generally leads to inefficient genome packaging (Fig. 4c, d).

\section{Differences in genome packaging efficiencies between mammalian and insect cells}

Based on the different vRNA:infectivity ratios we found between mammalian and insect cells (Fig. 1h, i), we aimed to further evaluate potential host cell differences in genome packaging using our vRNA FISH-immunofluorescence method on RVFV infected insect cells. Although we managed to visualize RVFV virions and vRNPs in insect cells (Supplementary Fig. 5), the image acquisition and analysis process at single-molecule resolution proved to be very challenging due to the elongated distribution in the z-axis of virion assembly sites. As an alternative, we applied our method to immobilized virions from virus stocks produced in different host cells and compared the genome composition of their virions (Fig. 5a-c). In general, virus stocks consist of a heterogeneous population of empty virions and virions with one, two or three genome segments (Fig. 5d). Interestingly, in mammalian cells (Vero E6) the S segment was packaged more often than the $\mathrm{M}$ and L segments, whereas in insect cells (C6/36) we observed the opposite (Fig. 5e). Consistent with the analysis of newly formed virions (Fig. 4a) and our own previous report ${ }^{27}$, about $50 \%$ of total RVFV particles produced on 
Vero E6 cells were empty. On the other hand, empty particles of virus stocks produced on C6/36 cells accounted for a considerably lower fraction (approximately $30 \%$ of total virions), indicating that despite bunyavirus genome packaging seems to be a largely stochastic process, the incorporation of genome segments into virions occurs more efficiently in insect cells than in mammalian cells. In addition, in insect cells the three different genome segments were incorporated into the same virion around 3 times more often than in mammalian cells ( 23\% versus $\sim 7 \%$ ) (Fig. 5d), generating a higher percentage of complete particles and showing an overall more efficient genome packaging process.

\section{Visualization of viral complementary RNAs incorporated into newly formed progeny virions}

Previous reports have found viral antigenomes, together with mRNA transcripts here referred to as viral complementary RNAs (cRNAs), in supernatants of bunyavirus infected cells or in purified virions preparations, as evidence for their incorporation into virus particles ${ }^{26,30-32}$. Here, we designed FISH probe sets to specifically recognize the cRNAs of RVFV and directly visualized their packaging using the vRNA FISH-immunofluorescence method (Fig. 6a, Supplementary Fig. 6 and Supplementary Table 1). Due to a maximum capacity to properly filter light wavelengths up to 5 different channels, we assessed the packaging of one viral segment and the corresponding cRNA in pairs. Indeed, all three RVFV cRNAs were occasionally incorporated into virions (Fig. 6b). Interestingly, we again observed high cell-to-cell variability in packaging efficiency within the cell populations. Furthermore, the ratios between the frequencies of incorporation of the viral genomes and the respective cRNAs differed per segment, resulting in ratios of approximately 4:1, 9:1 and 14:1 for S/cS, M/cM and L/cL, respectively (Fig. 6c). Although packaging of cRNAs occurs less frequently than that of viral genome segments, the direct visualization of virions containing cRNAs provides additional evidence of the absence of a selective mechanism that favors exclusively the incorporation of viral genome segments.

\section{Discussion}

The molecular mechanisms involved in the production of infectious bunyavirus progeny are yet to be discovered. Remarkably little is known about the principles that drive the genome packaging process of the multi-segmented bunyavirus genome into virions. Here, we combined smFISH and immunofluorescence assays to determine the genomic composition of RVFV and SBV virions at single-particle resolution by simultaneous detection of individual virus particles and vRNPs (Figs. 2, 3 and Supplementary Figs. 3, 4). Notably, we were able to link the intracellular abundance of specific vRNPs with the composition of progeny virions in individual infected cells and were able to show striking differences between genome packaging efficiencies in mammalian and insect cells.

By analyzing individual infected cells and their progeny virions, we not only observed a high cell-to-cell variability in packaging efficiency, which leads to a highly diverse composition of the progeny virion population, but also learned that the relative intracellular abundance of the vRNPs can influence, at least 
partially, overall genome packaging efficiencies (Fig. 4a). Our observations, obtained from single-cell analysis, are consistent with previous reports on purified virions of RVFV studied at a population level by Northern blotting, which suggested that the relative abundance of each genome segment in virions roughly approximated their relative abundances intracellularly ${ }^{33,34}$. Likewise, we found that a low relative intracellular abundance of a particular genome segment correlates with a low packaging frequency of that segment and vice versa. Accordingly, when the overall intracellular vRNP content was imbalanced (i.e. S:M:L ratio moved away from the theoretical 1:1:1 ratio), virions produced from that cell rarely contained the three genome segments and packaging was most likely very inefficient (Fig. 4b). On the other hand, a balanced intracellular vRNP content appears to serve as an essential precondition for the generation of complete particles, although it does not ensure in all cases an overall efficient genome packaging (Fig. 4c, d).

Contrary to other segmented RNA viruses like influenza virus and rotavirus, in which specific RNA-RNA interactions facilitate co-packaging of all the different viral genome segments ${ }^{20,35,36}$, a growing body of evidence supports the notion that bunyavirus genome packaging is rather flexible and non-selective ${ }^{21}$. Here, we show that less than $10 \%$ of RVFV and SBV progeny virions produced in mammalian cells contain the three genome segments, meaning that only a minor fraction of produced virus particles are infectious on their own (Fig. 4a). These results are in line with our previous report ${ }^{27}$, which suggested that bunyavirus genome packaging occurs without a specific mechanism that guarantees a consistent incorporation of all three genome segments into the same particle. In addition, we showed that the incorporation of S, M and L cRNAs into virions does occur, but not frequently. Importantly, packaging of cRNAs occurs disregarding whether the corresponding vRNA segment has or not an ambisense coding strategy (Fig. 6b, c). Although we observed similar non-selective features regarding genome packaging of tri-segmented bunyaviruses that belong to two different families, the low particle-to-PFU ratios previously reported for $\mathrm{BUNV}^{22}$ and Crimean-Congo Hemorrhagic fever virus $^{37}$ (family Nairoviridae, genus Orthonairovirus) imply that other bunyavirus species may have evolved towards a more efficient packaging process, but this remains to be studied.

Phleboviruses and orthobunyaviruses sustain a life cycle characterized by alternating productive infections between vertebrates and arthropod vectors ${ }^{4}$, underscoring the importance of studying the virus biology in both hosts. In an experimental infection study in goats, the source of the virus was found to cause differences in the course of infection. Insect cell-derived RVFV appeared to be more infectious than mammalian cell-derived RVFV based on faster peak viremia, infection of peripheral blood mononuclear cells, induction of fever and cytokine levels ${ }^{38}$. From our in vitro virus replication experiments, we noticed that insect cells required fewer genome equivalents per infectious unit compared to mammalian cells (Fig. 1h, i). Furthermore, we found that in RVFV progeny derived from insect cells, the relative amount of particles containing a full set of genome segments were about three 
times more compared to mature RVFV virions produced in mammalian cells (Fig. 5d). These observations strongly suggest that genome packaging occurs more efficiently in insect cells, which possibly contributes to maintain high viral loads during replication in the arthropod vector to enable efficient transmission to vertebrates. The reasons behind the more efficient genome packaging in insect cells are yet unknown, but could be related to the evolutionary origin of the viruses, which has been suggested to be of arthropod-specific ancestors ${ }^{39}$. In addition to host differences, the fact that the vRNA:infectivity ratio in plasma samples from experimentally infected lambs increased over time indicates that genome packaging efficiency may vary within a single host over the course of infection (Fig. 1m).

Interestingly, the bunyavirus genome packaging process investigated here gives rise to a large fraction of incomplete virus particles lacking one or two genome segments (Figs. 4a, 5d). Recently, a study with an influenza virus strictly dependent on genome complementation by co-infection demonstrated that incomplete influenza virus particles contributed to localized within-host spread ${ }^{40}$. This raises the intriguing question of whether co-infection by complementing incomplete particles may compensate for the inefficiency observed in bunyavirus genome packaging. In this hypothetical scenario, where complete particles are dispensable for a productive infection, bunyaviruses may resemble the life cycle of multi-partite viruses, which establish a productive infection by independent transmission of a complementary ensemble of particles each containing a single genome segment ${ }^{41,42}$.

Besides the potential role that incomplete particles may play in dissemination of bunyaviruses, additional strategies that would increase the flexibility also seem plausible as ways to overcome the bottleneck of an overall inefficient genome packaging process. Incorporating more than three genome segments per particle increases the probability of packaging at least one copy of S, M and L segments. Cryo-electron microscopy analyses of RVFV particles ${ }^{43,44}$ suggest that additional genome segments would fit within the intra-virion space. Another potential strategy involves the transmission of a large number of virions in structures known as collective infectious units, which result in a locally increased multiplicity of infection (MOI) ${ }^{45}$. It should also be noted, that a flexible packaging process may actually be best suited for the changing environments faced by the virus during its life cycle between vertebrates and arthropods. Finally, flexible packaging capabilities in terms of non-selectivity towards specific RNA sequences also facilitate the occurrence of reassortment events with related viruses, which increases genetic diversity and favors virus evolution.

In summary, here we studied genome replication and packaging of prototype bunyaviruses in mammalian and insect cells, both at a single-particle and single-cell level, as well as at a virion population and cell population level. Taken together, the evidence presented in this report further 
demonstrates that packaging of bunyavirus genome segments is a flexible, non-selective process and that genome packaging is more efficient in insect cells compared to mammalian cells.

\section{Methods}

\section{Cell lines}

Vero E6 cells (ATCC CRL-1586) were maintained in minimum essential medium (MEM) supplemented with $5 \%$ fetal bovine serum (FBS), $1 \%$ antibiotic/antimycotic, $1 \%$ MEM non-essential amino acids (MEM NEAA) and $2 \mathrm{mM} \mathrm{L-glutamine} \mathrm{at} 37^{\circ} \mathrm{C}$ and $5 \% \mathrm{CO}_{2}$. C6/36 cells (ATCC CRL-1660) were maintained in L-15 medium (Leibovitz) (Sigma-Aldrich) supplemented with $10 \% \mathrm{FBS}$, $1 \%$ antibiotic/antimycotic, $1 \%$ MEM NEAA and $2 \%$ tryptose phosphate broth at $28^{\circ} \mathrm{C}$. KC cells were maintained in Schneider's Drosophila medium supplemented with 10\% FBS and $1 \%$ antibiotic/antimycotic at $28^{\circ} \mathrm{C}$. Cell culture media and supplements were purchased from Gibco, unless specified otherwise.

\section{Viruses}

Virus stocks of RVFV strain Clone $13^{46}$ were obtained after infection of Vero E6 or C6/36 cells at a MOI of 0.005. Virus stocks of SBV isolate NL-F6 ${ }^{47}$ were obtained after infection of Vero E6 cells at a MOI of 0.01 .

\section{Genome segment-specific quantitative RT-PCR}

Mammalian cells (Vero E6) or insect cells (C6/36 for RVFV and KC for SBV) were seeded in 6-well cell culture plates at $2 \times 10^{5}$ cells/well or $6 \times 10^{5}$ cells/well, respectively, and allowed to attach for 2-4 h. Cells were subsequently infected at a MOI of 0.01 and after incubation for $3.5 \mathrm{~h}$, the inoculum was removed and substituted with fresh medium. At defined time points (varied per experiment), samples from the culture supernatant and cells were collected. In addition to the in vitro experiments, plasma samples were obtained from another study (lambs \#158, \#160 and \#162) in which lambs were experimentally infected via intravenous route with a $10^{5} \mathrm{TCID}_{50}$ dose of RVFV strain $35 / 74^{28}$.

From 1-2 mL of cell lysate, $200 \mu \mathrm{L}$ of culture supernatant or $200 \mu \mathrm{L}$ of plasma, total nucleic acid extractions were performed with the NucliSENS easyMAG system (bioMérieux) according to the instructions of the manufacturer. Subsequently, viral cDNA was synthesized with the SuperScript IV First-Strand Synthesis System for RT-PCR (Invitrogen) using a combination of S, M and L segment-specific primers (Supplementary Table 2), according to the instructions of the manufacturer. After the reverse transcription reaction, quantitative PCR amplifications were performed with the Power SYBR Green PCR Master Mix using $5 \mu \mathrm{L}$ of 20- or 200 -fold diluted cDNA preparations in a total volume of $25 \mu \mathrm{L}$, in combination with a 7500 Fast Real-Time PCR System (Applied Biosystems). Fragments from each segment were amplified using specific primers (Supplementary Table 3) under the 
following conditions: an initial denaturation step at $95^{\circ} \mathrm{C}$ for $10 \mathrm{~min} ; 40$ cycles of denaturation at $95^{\circ} \mathrm{C}$ for $15 \mathrm{~s}$, annealing at $59^{\circ} \mathrm{C}$ for $30 \mathrm{~s}$ and extension at $72^{\circ} \mathrm{C}$ for $36 \mathrm{~s}$; and a single cycle of denaturation at $95^{\circ} \mathrm{C}$ for $15 \mathrm{~s}$, annealing at $60^{\circ} \mathrm{C}$ for $1 \mathrm{~min}$, denaturation at $95^{\circ} \mathrm{C}$ for $15 \mathrm{~s}$ and annealing at $60^{\circ} \mathrm{C}$ for $15 \mathrm{~s}$. Data were acquired and analysed with the 7500 Fast System software version 1.5.1. (Applied Biosystems). Genome copies of each viral segment were finally calculated by intrapolation of the respective standard curve prepared with 10-fold serial dilutions of the viral segment cloned in pUC57 plasmids starting at $0.1 \mathrm{ng} / \mu \mathrm{L}$.

\section{Virus titration}

Infectious virus titers of samples from the in vitro replication experiments were determined with an immunoperoxidase monolayer assay. Vero E6 cells $\left(2 \times 10^{4}\right.$ cells/well) were incubated with 10 -fold serial dilutions (starting at 1:10) of cell culture supernatants for $72 \mathrm{~h}$ at $37^{\circ} \mathrm{C}$ and $5 \% \mathrm{CO}_{2}$. After incubation, cells were fixed with $4 \%$ paraformaldehyde for $15 \mathrm{~min}$, washed with PBS supplemented with $0.5 \%$ Tween 80 (PBST), and permeabilized with $1 \%$ Triton X-100 in PBS for 5 min. Next, samples were blocked with $100 \mu \mathrm{L} /$ well of $5 \%$ horse serum in PBS and subsequently incubated in sequential steps with $100 \mu \mathrm{L} /$ well of primary and secondary antibodies. Hybridoma 4-D4 ${ }^{48}$ supernatant (1:40 dilution) and serum from an experimentally infected sheep (1:1,000 dilution), were used as primary antibodies against RVFV and SBV, respectively. As secondary antibodies, HRP-conjugated rabbit polyclonal anti-mouse immunoglobulins (1:500 dilution, Dako) and HRP-conjugated rabbit polyclonal anti-sheep IgG (1:500 dilution, ab6747 Abcam) were used. Incubations with the blocking solution, primary and secondary antibodies were each for $1 \mathrm{~h}$ at $37^{\circ} \mathrm{C}$. Plates were washed with PBST between the addition of primary and secondary antibodies. For staining, $100 \mu \mathrm{L} /$ well of a $0.2 \mathrm{mg} / \mathrm{mL}$ amino ethyl carbazole solution in $500 \mathrm{mM}$ acetate buffer $\mathrm{pH} 5.0,88 \mathrm{mM} \mathrm{H}_{2} \mathrm{O}_{2}$ was added as substrate. Samples were analyzed in triplicate and the titer calculated as the median tissue culture infectious dose $\left(\mathrm{TCID}_{50} / \mathrm{mL}\right)$ using the Spearman-Kärber method. Virus titers of plasma samples were determined with a virus isolation assay as reported ${ }^{28}$.

\section{Single-molecule RNA FISH-immunofluorescence}

Experiments were performed with slight modifications to the Stellaris protocol for simultaneous FISHimmunofluorescence in adherent cells (Biosearch Technologies) ${ }^{49-51}$. Vero E6 cells $\left(1.5 \times 10^{4}\right.$ cells/well) or C6/36 cells $\left(4.5 \times 10^{4}\right.$ cells/well) were seeded on CultureWell 16 removable chambered coverglass (Grace Bio-Labs). Following overnight incubation at $37^{\circ} \mathrm{C}$ and $5 \% \mathrm{CO}_{2}$ (Vero E6) or $28^{\circ} \mathrm{C}(\mathrm{C} 6 / 36)$, cells were infected with RVFV or SBV at MOIs of 0.33-1.00. One hour post-infection the medium was refreshed. At defined time points (varied per experiment), cells were fixed and permeabilized with a 3:1 mixture of methanol (Merck) - glacial acetic acid (Merck) for $10 \mathrm{~min}$. Cells were subsequently washed twice with PBS and once with pre-hybridization buffer (10\% deionized formamide [Millipore] in 2x concentrated SSC [Gibco]) for $5 \mathrm{~min}$. Cells were then 
incubated for $12-16 \mathrm{~h}$ at $37^{\circ} \mathrm{C}$ with $100 \mu \mathrm{L} /$ well of virus-specific FISH probe sets (Supplementary Table 1) and primary antibodies in hybridization buffer (10\% deionized formamide, $10 \%$ dextran sulphate [Sigma-Aldrich], $2 \mathrm{mM}$ vanadyl ribonucleoside complexes [VRC, Sigma-Aldrich] in 2x SSC). Custom probe sets were designed using the RNA FISH Probe Designer tool (https://www.biosearchtech.com/support/tools/design-software/stellaris-probe-designer) and purchased from Biosearch Technologies (Petaluma, California and Risskov, Denmark). FISH probes were added at a final concentration of $250 \mathrm{nM}$ for RVFV and $125 \mathrm{nM}$ for SBV. Hybridoma 4-D4 ${ }^{48}$ supernatant (1:160 dilution) and serum from an immunized rabbit $^{52}$ (1:4,000 dilution), were used as primary antibodies against RVFV and SBV, respectively. Following hybridization and incubation with primary antibodies, cells were extensively washed at $37^{\circ} \mathrm{C}$ (twice with pre-hybridization buffer for $30 \mathrm{~min}$ and twice with 2x SSC for $15 \mathrm{~min}$ ). Subsequently, cells were incubated with $100 \mu \mathrm{L} /$ well of secondary antibodies for $1 \mathrm{~h}$ at $37^{\circ} \mathrm{C}$. A goat polyclonal anti-mouse IgG labelled with Alexa Fluor 488 (1:1,000 dilution, A-11001 Invitrogen) or a goat polyclonal anti-rabbit IgG labelled with FITC (1:400 dilution, sc-2012 Santa Cruz Biotechnology) were used as secondary antibodies. Next, cells were washed twice with $2 \mathrm{x}$ SSC, and nuclei were stained by incubation with $100 \mu \mathrm{L} /$ well of $1 \mu \mathrm{g} / \mathrm{mL}$ DAPI in 2x SSC for 5 min. Finally, cells were washed with 2x SSC and submerged in VectaShield antifade mounting medium H-1000 (Vector Laboratories). For analysis of virus stocks, $100 \mu \mathrm{L} /$ well of virus stocks diluted 1:3 were added on CultureWell 16 removable chambered coverglass and virions were allowed to attach to the surface for $5 \mathrm{~h}$ at $28^{\circ} \mathrm{C}$. From the fixation step onwards, the same procedure as described for adherent cells was followed. The specificity of the FISH probes and antibodies was confirmed with single-color controls (Supplementary Figs. 1, 2, 6). Mock-infected samples and samples without primary antibodies were used as negative controls.

\section{Image acquisition and analysis}

Z-stacked images of infected cells and immobilized virions, with a fixed interval of $0.28-0.31 \mu \mathrm{m}$ between slices, were acquired with an inverted widefield fluorescence microscope Axio Observer 7 (ZEISS, Germany) using appropriate filters and a 1.3 NA 100x EC Plan-NEOFLUAR oil objective in combination with an AxioCam MRm CCD camera. Exposure times were defined empirically and differed depending on the cell line, probe sets and fluorescent dyes. Raw images were deconvolved in standard mode using Huygens Professional version 19.10 (Scientific Volume Imaging B.V., The Netherlands). If required, raw images were Z-aligned in ZEN 2.6 Pro (ZEISS, Germany) before deconvolution. For analysis, 3D data was converted to maximum intensity projections using Z-project within Image $\mathrm{J}^{53}$. Detection, quantification and co-localization analyses of individual spots, each representing a single virion, vRNP or cRNA, were performed in ImageJ in combination with the plugin ComDet version 0.5.0 (https://github.com/ekatrukha/ComDet). Spot detection thresholds for each channel were set empirically by individual examination of images. Intracellular genome composition analysis considered a region of the cytoplasm representative of the overall composition, not including 
the Golgi apparatus where signal is generally overcrowded due to vRNP accumulation (Fig. 3d and Supplementary Fig. 3). Genome composition analysis of progeny virions only considered virus particles in selected regions of interest located distant from the nucleus of the infected cell (Fig. 3e and Supplementary Fig. 3). For visualization purposes, image brightness and contrast were manually adjusted in ImageJ. Finally, Imaris 9.5 software (Bitplane, Switzerland) was utilized to create optimal $3 \mathrm{D}$ representations of the data using the Surface and Spots modes.

\section{Statistical analysis and data visualization}

Prism 8 (GraphPad Software) was used to generate graphs and perform statistical analysis. Mean vRNA:infectivity ratios were compared using an unpaired two-tailed Student's $t$ test with Welch's correction (not assuming equal variances). The correlation between the intracellular vRNP relative frequency and packaged vRNP relative frequency was calculated with the Pearson's correlation coefficient (r). $p$ values $\geq 0.05$ were considered not significant.

\section{Data availability}

The authors declare that the data supporting the findings of this study are available within the paper and its supplementary information files.

\section{References}

1. Walter, C. T. \& Barr, J. N. Recent advances in the molecular and cellular biology of bunyaviruses. Journal of General Virology, 92, 2467-2484 (2011).

2. Hughes, H. R. et al. ICTV Virus Taxonomy Profile: Peribunyaviridae. Journal of General Virology, 101, 1-2 (2020).

3. Abudurexiti, A. et al. Taxonomy of the order Bunyavirales: update 2019. Arch Virol 164, 1949-1965 (2019).

4. Horne, K. M. \& Vanlandingham, D. L. Bunyavirus-Vector Interactions. Viruses 6, 4373-4397 (2014).

5. Elliott, R. M. \& Brennan, B. Emerging phleboviruses. Current Opinion in Virology 5, 50-57 (2014).

6. Elliott, R. M. Orthobunyaviruses: recent genetic and structural insights. Nature Reviews Microbiology 12, 673685 (2014).

7. Elliott, R. M. Molecular biology of the Bunyaviridae. Journal of General Virology, 71, 501-522 (1990).

8. Guardado-Calvo, P. \& Rey, F. A. Chapter Three - The Envelope Proteins of the Bunyavirales. in Advances in Virus Research (eds. Kielian, M., Mettenleiter, T. C. \& Roossinck, M. J.) vol. 98 83-118 (Academic Press, 2017). 
9. Överby, A. K., Pettersson, R. F., Grünewald, K. \& Huiskonen, J. T. Insights into bunyavirus architecture from electron cryotomography of Uukuniemi virus. PNAS 105, 2375-2379 (2008).

10.Freiberg, A. N., Sherman, M. B., Morais, M. C., Holbrook, M. R. \& Watowich, S. J. Three-Dimensional Organization of Rift Valley Fever Virus Revealed by Cryoelectron Tomography. Journal of Virology 82 , $10341-10348$ (2008).

11.Bowden, T. A. et al. Orthobunyavirus Ultrastructure and the Curious Tripodal Glycoprotein Spike. PLoS Pathogens 9, e1003374 (2013).

12.Briese, T., Calisher, C. H. \& Higgs, S. Viruses of the family Bunyaviridae: Are all available isolates reassortants? Virology 446, 207-216 (2013).

13.Gerber, M., Isel, C., Moules, V. \& Marquet, R. Selective packaging of the influenza A genome and consequences for genetic reassortment. Trends in Microbiology 22, 446-455 (2014).

14.Lakdawala, S. S., Fodor, E. \& Subbarao, K. Moving On Out: Transport and Packaging of Influenza Viral RNA into Virions. Annual Review of Virology 3, 411-427 (2016).

15.Borodavka, A., Desselberger, U. \& Patton, J. T. Genome packaging in multi-segmented dsRNA viruses: distinct mechanisms with similar outcomes. Current Opinion in Virology 33, 106-112 (2018).

16.Noda, T. et al. Architecture of ribonucleoprotein complexes in influenza A virus particles. Nature 439, 490492 (2006).

17.Noda, T. et al. Three-dimensional analysis of ribonucleoprotein complexes in influenza A virus. Nature Communications 3, 1-6 (2012).

18. Fournier, E. et al. A supramolecular assembly formed by influenza A virus genomic RNA segments. Nucleic Acids Res 40, 2197-2209 (2012).

19.Goto, H., Muramoto, Y., Noda, T. \& Kawaoka, Y. The Genome-Packaging Signal of the Influenza A Virus Genome Comprises a Genome Incorporation Signal and a Genome-Bundling Signal. Journal of Virology 87, $11316-11322$ (2013).

20.Borodavka, A., Dykeman, E. C., Schrimpf, W. \& Lamb, D. C. Protein-mediated RNA folding governs sequence-specific interactions between rotavirus genome segments. eLife 6, e27453 (2017).

21.Wichgers Schreur, P. J., Kormelink, R. \& Kortekaas, J. Genome packaging of the Bunyavirales. Current Opinion in Virology 33, 151-155 (2018).

22.Lowen, A. C., Boyd, A., Fazakerley, J. K. \& Elliott, R. M. Attenuation of Bunyavirus Replication by Rearrangement of Viral Coding and Noncoding Sequences. Journal of Virology 79, 6940-6946 (2005). 
23.Brennan, B., Welch, S. R., McLees, A. \& Elliott, R. M. Creation of a Recombinant Rift Valley Fever Virus with a Two-Segmented Genome. Journal of Virology 85, 10310-10318 (2011).

24. Kortekaas, J. et al. Creation of a Nonspreading Rift Valley Fever Virus. Journal of Virology 85, 12622-12630 (2011).

25.Wichgers Schreur, P. J., Oreshkova, N., Moormann, R. J. M. \& Kortekaas, J. Creation of Rift Valley Fever Viruses with Four-Segmented Genomes Reveals Flexibility in Bunyavirus Genome Packaging. Journal of Virology 88, 10883-10893 (2014).

26.Brennan, B., Welch, S. R. \& Elliott, R. M. The Consequences of Reconfiguring the Ambisense S Genome Segment of Rift Valley Fever Virus on Viral Replication in Mammalian and Mosquito Cells and for Genome Packaging. PLoS Pathogens 10, e1003922 (2014).

27. Wichgers Schreur, P. J. \& Kortekaas, J. Single-Molecule FISH Reveals Non-selective Packaging of Rift Valley Fever Virus Genome Segments. PLoS Pathogens 12, e1005800 (2016).

28. Wichgers Schreur, P. J. et al. Reproducing the Rift Valley fever virus mosquito-lamb-mosquito transmission cycle. Submitted for publication (2020).

29.Carnec, X., Ermonval, M., Kreher, F., Flamand, M. \& Bouloy, M. Role of the cytosolic tails of Rift Valley fever virus envelope glycoproteins in viral morphogenesis. Virology 448, 1-14 (2014).

30.Simons, J. F., Hellman, U. \& Pettersson, R. F. Uukuniemi virus S RNA segment: ambisense coding strategy, packaging of complementary strands into virions, and homology to members of the genus Phlebovirus. Journal of Virology 64, 247-255 (1990).

31.Kormelink, R., de Haan, P., Peters, D. \& Goldbach, R. Viral RNA synthesis in tomato spotted wilt virusinfected Nicotiana rustica plants. J. Gen. Virol. 73 ( Pt 3), 687-693 (1992).

32.Ikegami, T., Won, S., Peters, C. J. \& Makino, S. Rift Valley Fever Virus NSs mRNA Is Transcribed from an Incoming Anti-Viral-Sense S RNA Segment. Journal of Virology 79, 12106-12111 (2005).

33. Gauliard, N., Billecocq, A., Flick, R. \& Bouloy, M. Rift Valley fever virus noncoding regions of L, M and S segments regulate RNA synthesis. Virology 351, 170-179 (2006).

34.Murakami, S., Terasaki, K., Narayanan, K. \& Makino, S. Roles of the Coding and Noncoding Regions of Rift Valley Fever Virus RNA Genome Segments in Viral RNA Packaging. Journal of Virology 86, 4034-4039 (2012).

35.Dadonaite, B. et al. The structure of the influenza A virus genome. Nature Microbiology 4, 1781-1789 (2019). 
36.Le Sage, V. et al. Mapping of Influenza Virus RNA-RNA Interactions Reveals a Flexible Network. Cell Reports 31, (2020).

37. Weidmann, M. et al. Quantitative analysis of particles, genomes and infectious particles in supernatants of haemorrhagic fever virus cell cultures. Virology Journal 8, 81 (2011).

38.Nfon, C. K., Marszal, P., Zhang, S. \& Weingartl, H. M. Innate Immune Response to Rift Valley Fever Virus in Goats. PLoS Neglected Tropical Diseases 6, e1623 (2012).

39.Marklewitz, M., Zirkel, F., Kurth, A., Drosten, C. \& Junglen, S. Evolutionary and phenotypic analysis of live virus isolates suggests arthropod origin of a pathogenic RNA virus family. PNAS 112, 7536-7541 (2015).

40.Jacobs, N. T. et al. Incomplete influenza A virus genomes occur frequently but are readily complemented during localized viral spread. Nature Communications 10, 1-17 (2019).

41.Sicard, A., Michalakis, Y., Gutiérrez, S. \& Blanc, S. The Strange Lifestyle of Multipartite Viruses. PLoS Pathogens 12, e1005819 (2016).

42.Lucía-Sanz, A. \& Manrubia, S. Multipartite viruses: adaptive trick or evolutionary treat? npj Systems Biology and Applications 3, 1-11 (2017).

43.Huiskonen, J. T., Överby, A. K., Weber, F. \& Grünewald, K. Electron Cryo-Microscopy and Single-Particle Averaging of Rift Valley Fever Virus: Evidence for Gn-Gc Glycoprotein Heterodimers. Journal of Virology 83, 3762-3769 (2009).

44.Sherman, M. B., Freiberg, A. N., Holbrook, M. R. \& Watowich, S. J. Single-particle cryo-electron microscopy of Rift Valley fever virus. Virology 387, 11-15 (2009).

45.Sanjuán, R. Collective Infectious Units in Viruses. Trends in Microbiology 25, 402-412 (2017).

46. Muller, R. et al. Characterization of clone 13, a naturally attenuated avirulent isolate of Rift Valley fever virus, which is altered in the small segment. Am. J. Trop. Med. Hyg. 53, 405-411 (1995).

47.Hulst, M. et al. Genetic characterization of an atypical Schmallenberg virus isolated from the brain of a malformed lamb. Virus Genes 47, 505-514 (2013).

48. Keegan, K. \& Collett, M. S. Use of bacterial expression cloning to define the amino acid sequences of antigenic determinants on the G2 glycoprotein of Rift Valley fever virus. Journal of Virology 58, 263-270 (1986).

49.Femino, A. M., Fay, F. S., Fogarty, K. \& Singer, R. H. Visualization of Single RNA Transcripts in Situ. Science 280, 585-590 (1998).

50.Raj, A., van den Bogaard, P., Rifkin, S. A., van Oudenaarden, A. \& Tyagi, S. Imaging individual mRNA molecules using multiple singly labeled probes. Nature Methods 5, 877-879 (2008). 
51.Orjalo Jr., A., Johansson, H. E. \& Ruth, J. L. Stellaris ${ }^{\mathrm{TM}}$ fluorescence in situ hybridization (FISH) probes: a powerful tool for mRNA detection. Nature Methods $\mathbf{8 ,} 884$ (2011).

52.Oymans, J. et al. Reverse Genetics System for Shuni Virus, an Emerging Orthobunyavirus with Zoonotic Potential. Viruses 12, 455 (2020).

53.Schneider, C. A., Rasband, W. S. \& Eliceiri, K. W. NIH Image to ImageJ: 25 years of image analysis. Nature Methods 9, 671-675 (2012).

\section{Acknowledgements}

We thank Corinne Geertsema (Laboratory of Virology, Wageningen University) for kindly providing the KC cells, Michèle Bouloy (Institut Pasteur, France) for kindly providing the RVFV strain Clone 13, Eduardo de Freitas Costa and Jose L. Gonzalez (Department of Epidemiology, Bioinformatics and Animal Studies, Wageningen Bioveterinary Research) for advice on statistics, Hélène Cecilia (UMR 1300 BIOEPAR, INRAE/Oniris) and Quirine ten Bosch (Quantitative Veterinary Epidemiology, Wageningen University) for stimulating discussions, and Richard Kormelink (Laboratory of Virology, Wageningen University) for critically reviewing the manuscript and providing feedback. E.B.M. is a grateful recipient of scholarships from the Graduate School for Production Ecology \& Resource Conservation (PE\&RC) and Universidad de Costa Rica (OAICE-031-2019).

\section{Author contributions}

P.J.W.S. and J.K. conceived the project. C.M.S. contributed developing the 5-channel FISHimmunofluorescence method. E.B.M. and P.J.W.S. designed the experiments. E.B.M. performed the RT-qPCR, virus titration and FISH-immunofluorescence experiments. E.A.K. developed and customized the plugin ComDet for image analysis. E.B.M. and P.J.W.S. analyzed and interpreted the data with contributions of J.K. and E.A.K. P.J.W.S. and J.K. supervised the project. E.B.M and P.J.W.S. wrote the manuscript, prepared the figures and movies with contributions of J.K.

\section{Competing interests}

The authors declare no competing interests. 
a

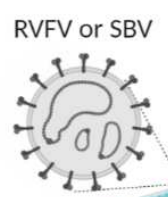

Infection of mammalian (Vero E6) or insect ( $\mathrm{C} 6 / 36$ or $\mathrm{KC})$ cells

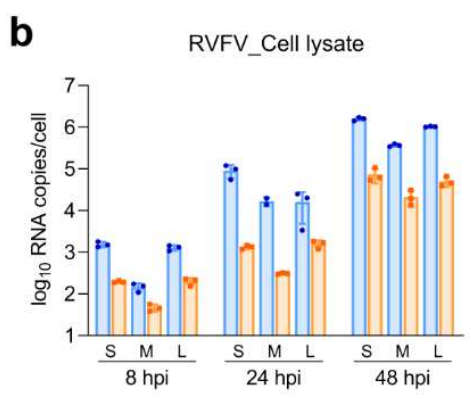

C

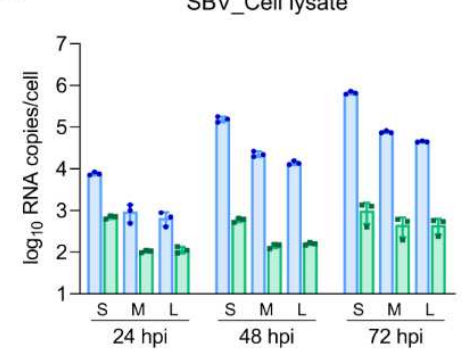

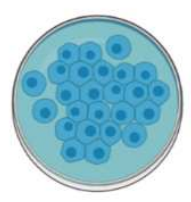

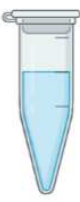

Collection of cell lysates and supernatants

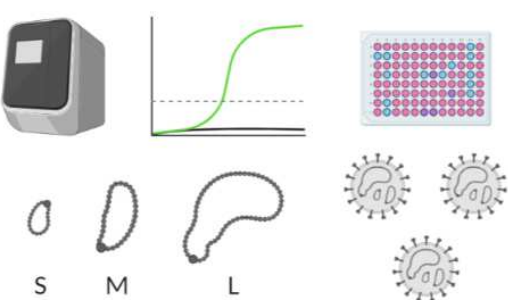

Quantification of genome segments and infectious virus d

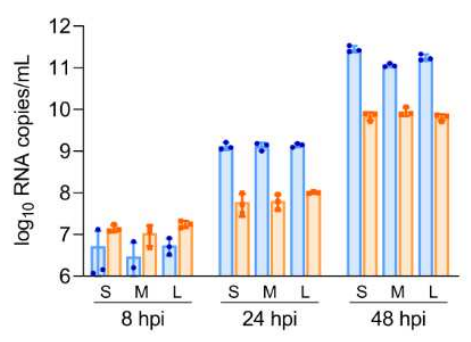

e

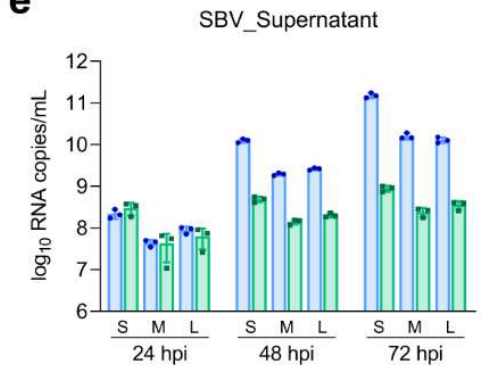

f

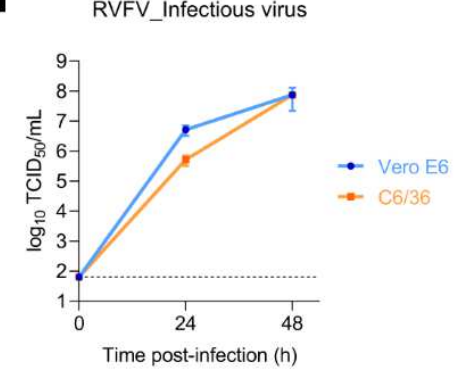

g

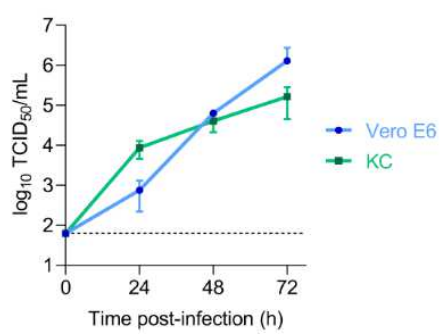

h

h RVFV_VRNA:infectivity ratio i

SBV_VRNA:infectivity ratio
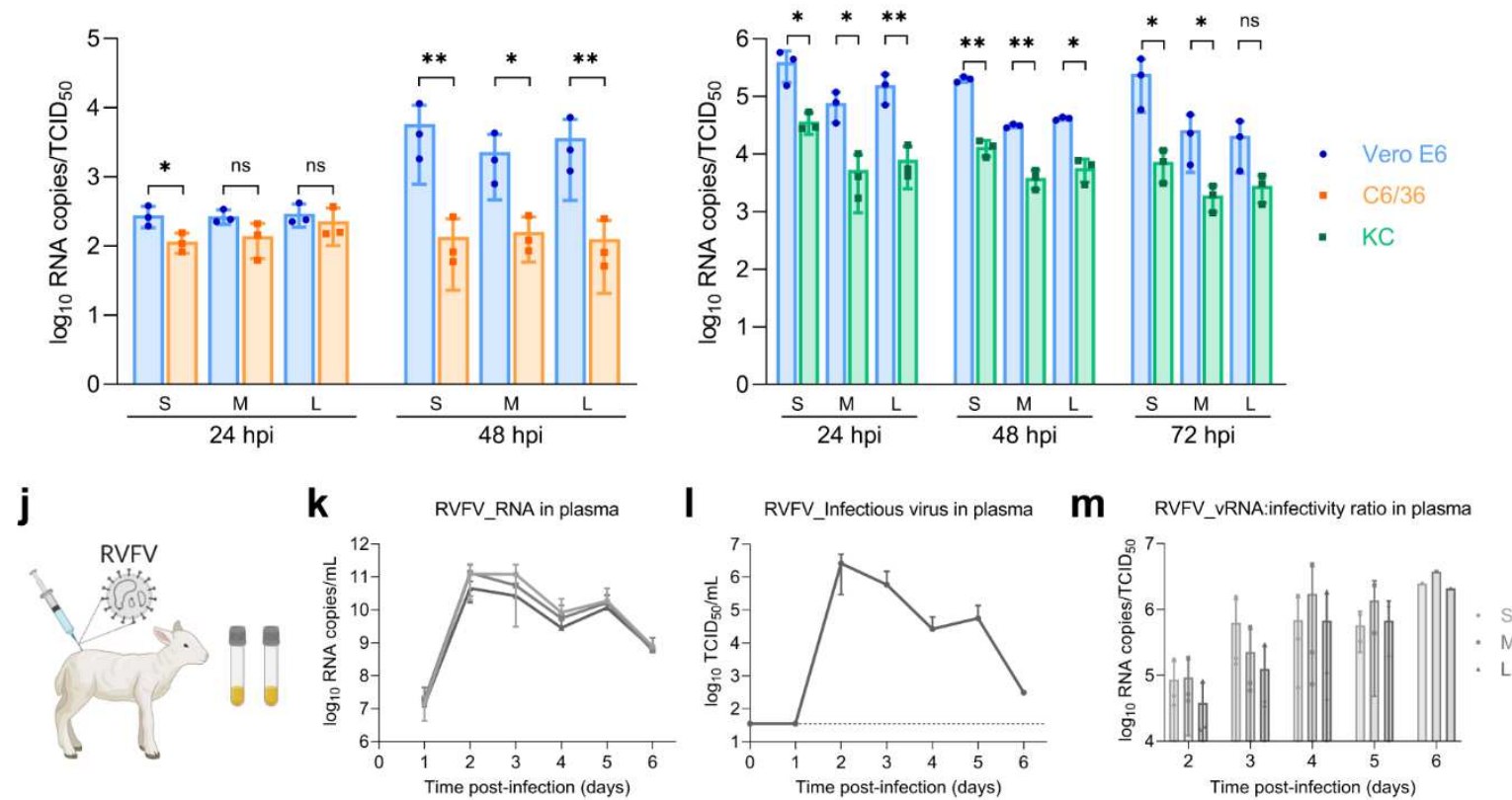

Fig. 1 Viral RNA:infectivity ratios in mammalian and insect hosts. a Schematic representation of the in vitro experimental setup. Mammalian (Vero E6) and insect (C6/36 and KC) cells were infected with RVFV or SBV 
586 (MOI 0.01). Cell lysates and supernatants were collected at defined time points. Viral RNA was quantified with

587 genome segment-specific RT-qPCRs and virus titers were determined by endpoint titration. b-e In vitro replication 588 kinetics of RVFV and SBV. Bars show means with SD. Dots represent biological replicates $\left(n=3\right.$ samples $\left.^{\dagger}\right)$. ${ }^{\dagger}$ Bar of 589 RVFV cell lysate M segment at $24 \mathrm{~h}$ post-infection shows mean of two samples. $\mathrm{f}, \mathrm{g}$ RVFV and SBV infectious titers 590 in cell culture supernatants. Titers correspond to the same supernatant samples analyzed in d, e. Graphs show 591 means with SD of $\mathrm{n}=3$ biological replicates. The dashed line indicates the limit of detection $\left(10^{1.80} \mathrm{TCID} 50 / \mathrm{mL}\right)$. $592 \mathrm{~h}, \mathrm{i}$ RVFV and SBV VRNA:infectivity ratios calculated as viral genome copies per infectious unit in cell culture 593 supernatants. Bars show means with SD. Dots represent individual ratios $(n=3)$. j Schematic representation of the 594 animal samples from another study obtained for analysis. Lambs were experimentally infected via intravenous 595 route with RVFV and plasma samples were collected daily ${ }^{28} . \mathrm{k}$ In vivo replication kinetics of RVFV. Graph shows 596 means with SD of plasma samples $\left(n=3^{\ddagger}\right)$ analyzed by RT-qPCR. I RVFV infectious titers in plasma as determined 597 with a virus isolation assay ${ }^{28}$. Graph shows means with SD of plasma samples $\left(n=3^{\ddagger}\right)$. The dashed line indicates 598 the limit of detection $\left(10^{1.55} \mathrm{TCID}_{50} / \mathrm{mL}\right)$. $\mathrm{m}$ RVFV in vivo VRNA:infectivity ratios calculated as viral genome copies 599 per infectious unit in plasma. Bars show means with SD. Dots represent individual ratios $\left(n=3^{\ddagger}\right)$. ${ }^{\ddagger}$ At early $(1$ day) 600 and late (5-6 days) times post-infection, genome copies and infectious titers of some samples were below the 601 limits of detection. In those cases, the reported values represent the mean of two samples or a single sample. 602 Statistical analysis of vRNA:infectivity ratios was performed using an unpaired two-tailed Student's $t$ test with Welch's correction (not assuming equal variances). ${ }^{*} p<0.05 ;{ }^{* *} p<0.01 ;$ ns, not significant $(p \geq 0.05)$. 
a

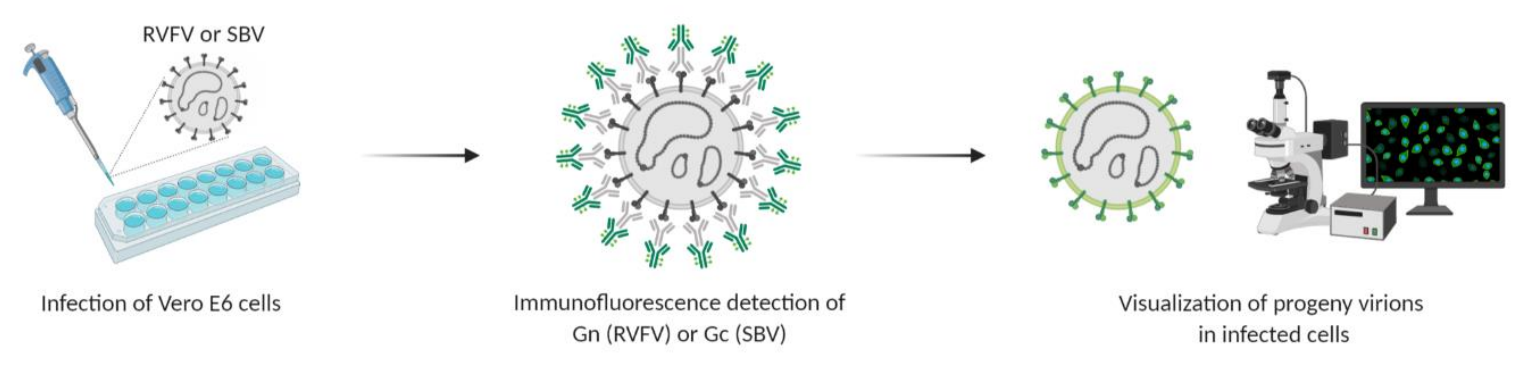

b
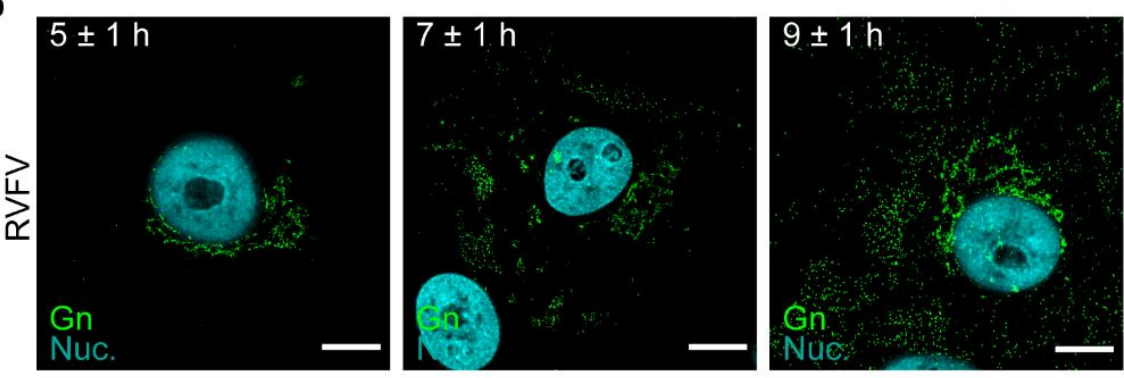

d
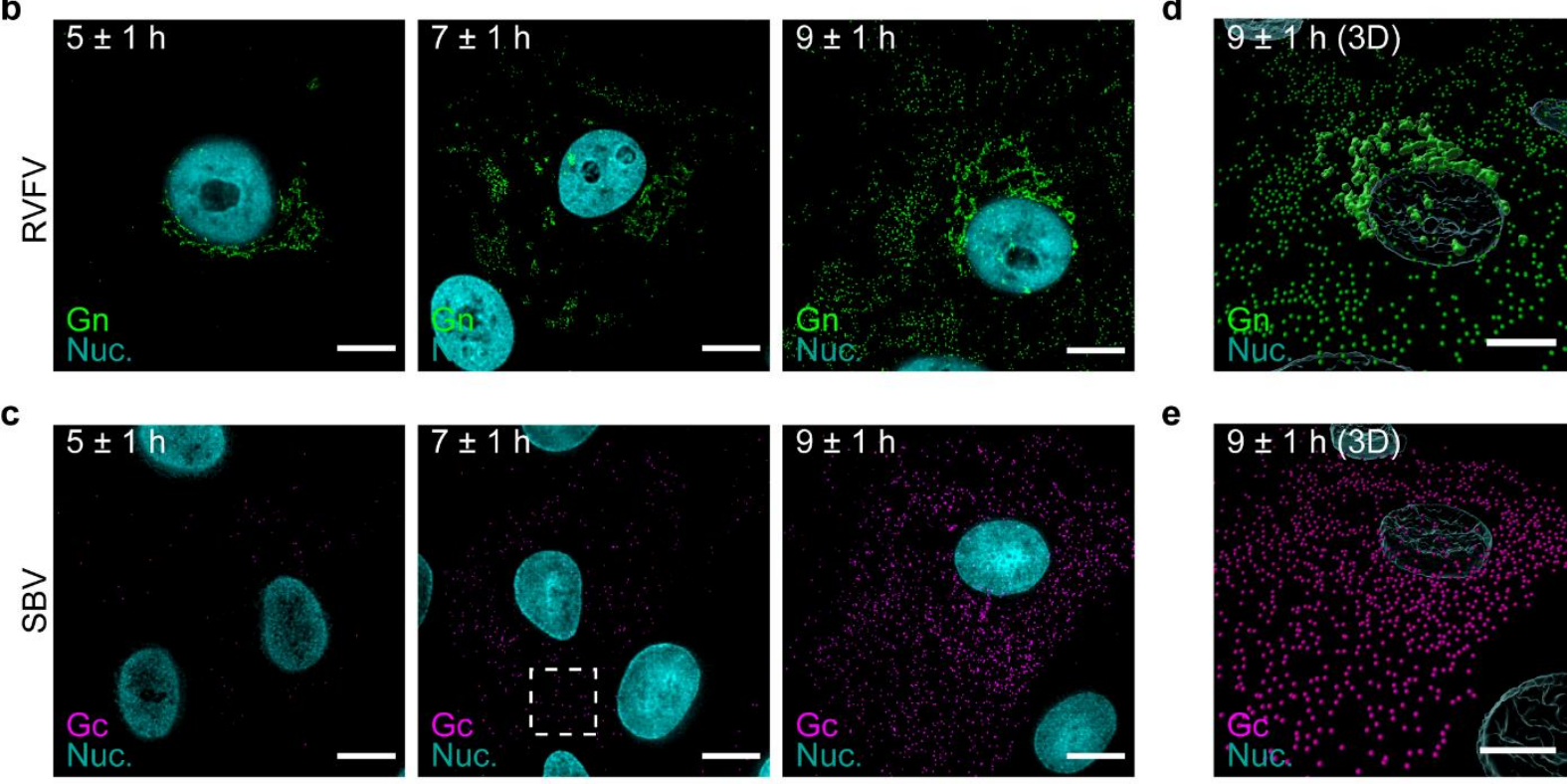

e

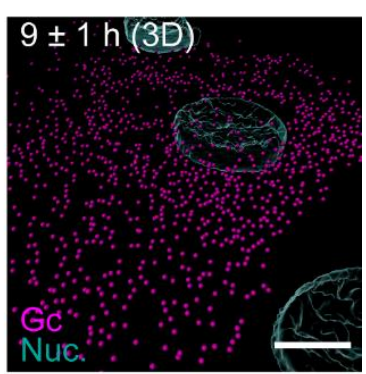

f
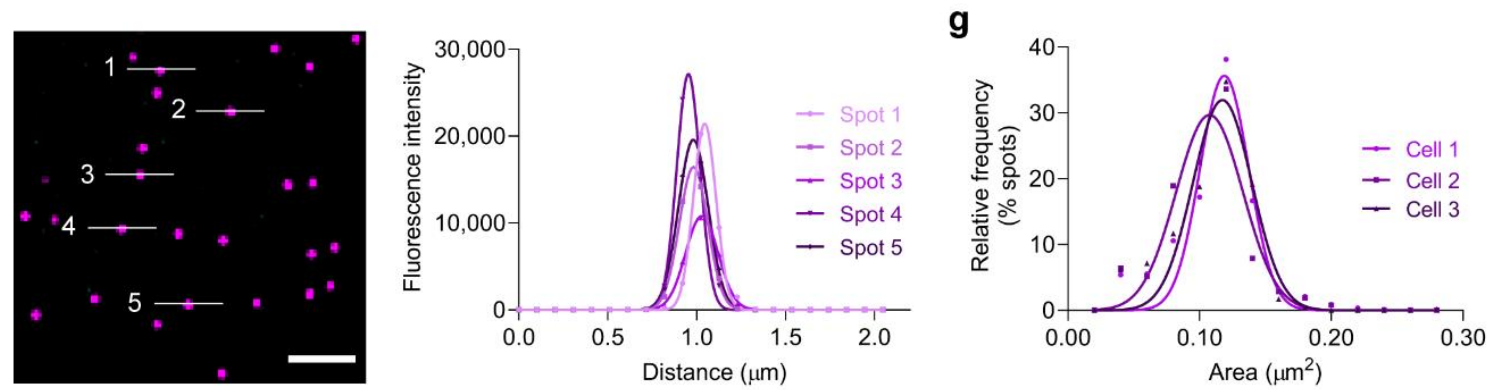

Fig. 2 Immunofluorescence detection of newly formed bunyavirus progeny virions at single-particle resolution. a Schematic representation of the experimental setup. Vero E6 cells were infected with RVFV (MOI 1) or SBV ( $\mathrm{MOI} 0.33$ ) and cells were fixed at defined time points. Progeny virions were detected by immunofluorescence. b, c Release kinetics of RVFV particles (green) (b) and SBV particles (magenta) (c). RVFV virions were detected with antibody 4-D4 ${ }^{48}$ targeting the $\mathrm{Gn}$ glycoprotein in combination with Alexa Fluor 488-conjugated secondary antibodies. SBV virions were detected with serum from an immunized rabbit ${ }^{52}$ targeting the Gc glycoprotein in combination with FITC-conjugated secondary antibodies. Cell nuclei (cyan) were visualized with DAPI. RVFV Gn accumulates in a perinuclear region, the site of virion assembly. $d$, e Three-dimensional representations showing the spatial distribution of virions at the $9 \pm 1 \mathrm{~h}$ time point created with Imaris using the Surfaces and Spots modes.

$614 \mathrm{f}$ Magnification of a region of interest (indicated as a dashed box in the second panel of c) and fluorescence intensity plot of the indicated spots. Dots represent data points and lines show Gaussian curves fitting the data. The unimodal distribution of fluorescence intensities along the lines crossing the spots is characteristic of single particles. $\mathbf{g}$ Histogram of the area of the spots detected in images of SBV infected cells ( $n=3$ cells; more than 500 
618 spots per image). Dots represent data points and lines show Gaussian curves fitting the data. The unimodal 619 distribution denotes reproducible measurements of single spots within and between images. Images are merged 620 maximum intensity projections of two channels. Scale bars, $10 \mu \mathrm{m}$ (b-e), $2 \mu \mathrm{m}(\mathbf{f})$. 


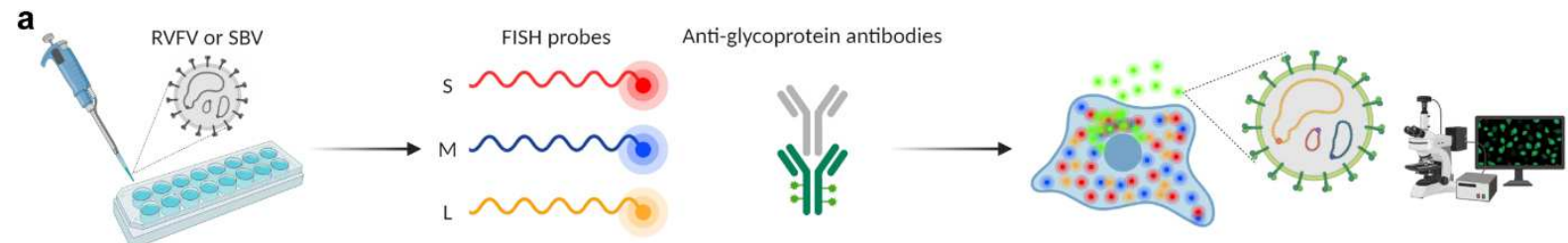

Infection of Vero E6 cells

smFISH-Immunofluorescence assay

Visualization of VRNPs and progeny virions in infected cells

b

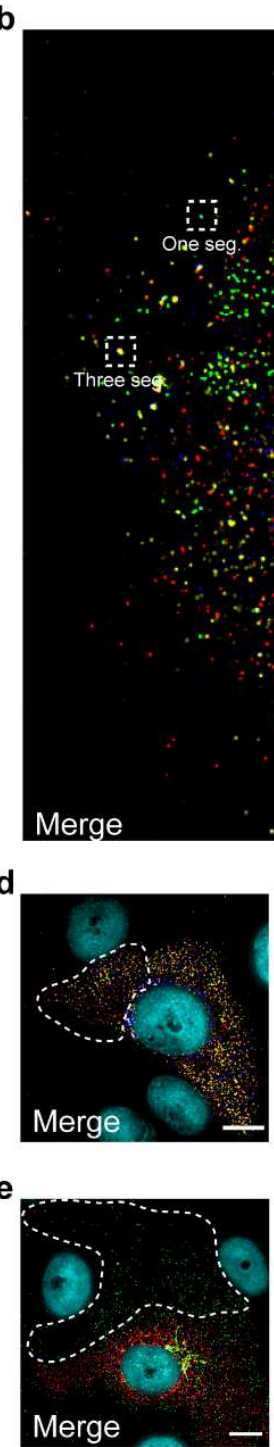

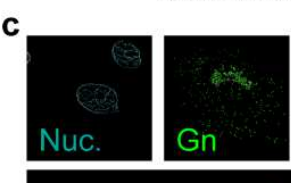
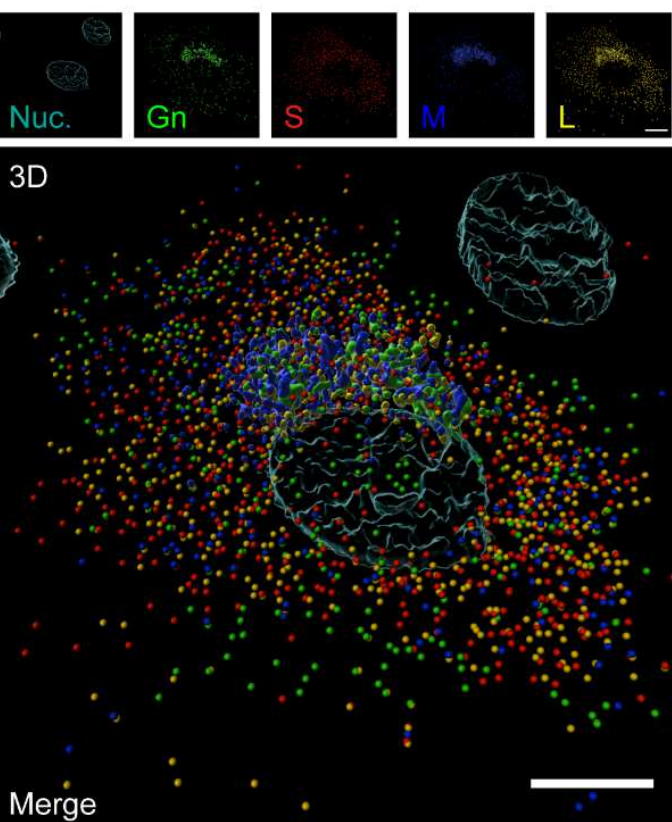

Merge
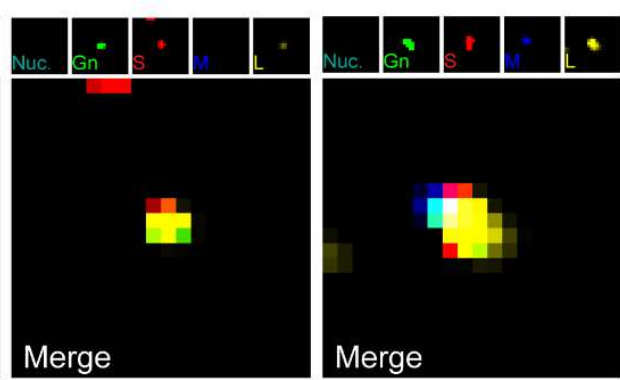

Merge

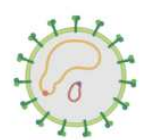

Two segments (SL)

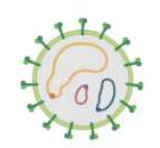

Three segments (SML)

Fig. 3 Single-molecule vRNA FISH-immunofluorescence of bunyavirus infected mammalian cells. a Schematic representation of the experimental setup. Vero E6 cells were infected with RVFV (MOI 0.50-0.75) or SBV ( $\mathrm{MOI} 0.33$ ) and cells were fixed at $8 \mathrm{~h}$ post-infection. The $\mathrm{S}$ segment ( $\mathrm{N}$ gene; red), $\mathrm{M}$ segment (polyprotein gene; blue) and L segment (RdRp gene; yellow) were hybridized using probe sets labelled with CAL Fluor Red 610, Quasar 670 and Quasar 570, respectively. Progeny RVFV particles (green) were detected with antibody 4-D448 targeting the Gn glycoprotein in combination with Alexa Fluor 488-conjugated secondary antibodies. Progeny SBV particles (Supplementary Figs. 3b, 4) were detected with serum from an immunized rabbit ${ }^{52}$ targeting the Gc glycoprotein in combination with FITC-conjugated secondary antibodies. Cell nuclei (cyan) were visualized with DAPI. Individual spots, each representing either a single VRNP or a virus particle were detected, counted and assessed for co-localization in ImageJ with the plugin ComDet. $\mathbf{b}$ Visualization of vRNPs and progeny virions of a 
632 RVFV infected cell. The dashed boxes highlight individual virus particles subjected to co-localization analysis for 633 example purposes. The number of RVFV genome segments in each highlighted particle is indicated. 634 c Three-dimensional representation showing the spatial distribution of vRNPs and virions of the image displayed 635 in b created with Imaris using the Surfaces and Spots modes. Accumulation of vRNPs and co-localization to the 636 same perinuclear region as Gn show active VRNP recruitment to the site of virion assembly. Co-localization of 637 VRNPs and virions is depicted by merged spheres. $d$, e RVFV infected cells. The dashed contours represent example 638 regions of interest selected for quantification of cytoplasmic VRNPs (d) and determining the genome composition 639 of extracellular virions through co-localization analysis (e) (Supplementary Fig. 3a). Example regions of interest 640 selected for analysis of SBV infected cells are shown in Supplementary Fig. 3b. $f$ Magnification of regions of interest 641 indicated by dashed boxes in $\mathbf{b}$. The genome composition of each virion can be deduced from the spots detected 642 on each individual channel. Images are merged maximum intensity projections of four (d) or five (b, c, e, f) 643 channels. Due to a higher fluorescence intensity of the green channel compared to the other channels, spots 644 co-localizing with the glycoprotein may sometimes appear masked and not entirely evident in merged images. 645 Scale bars, $10 \mu \mathrm{m}$. 

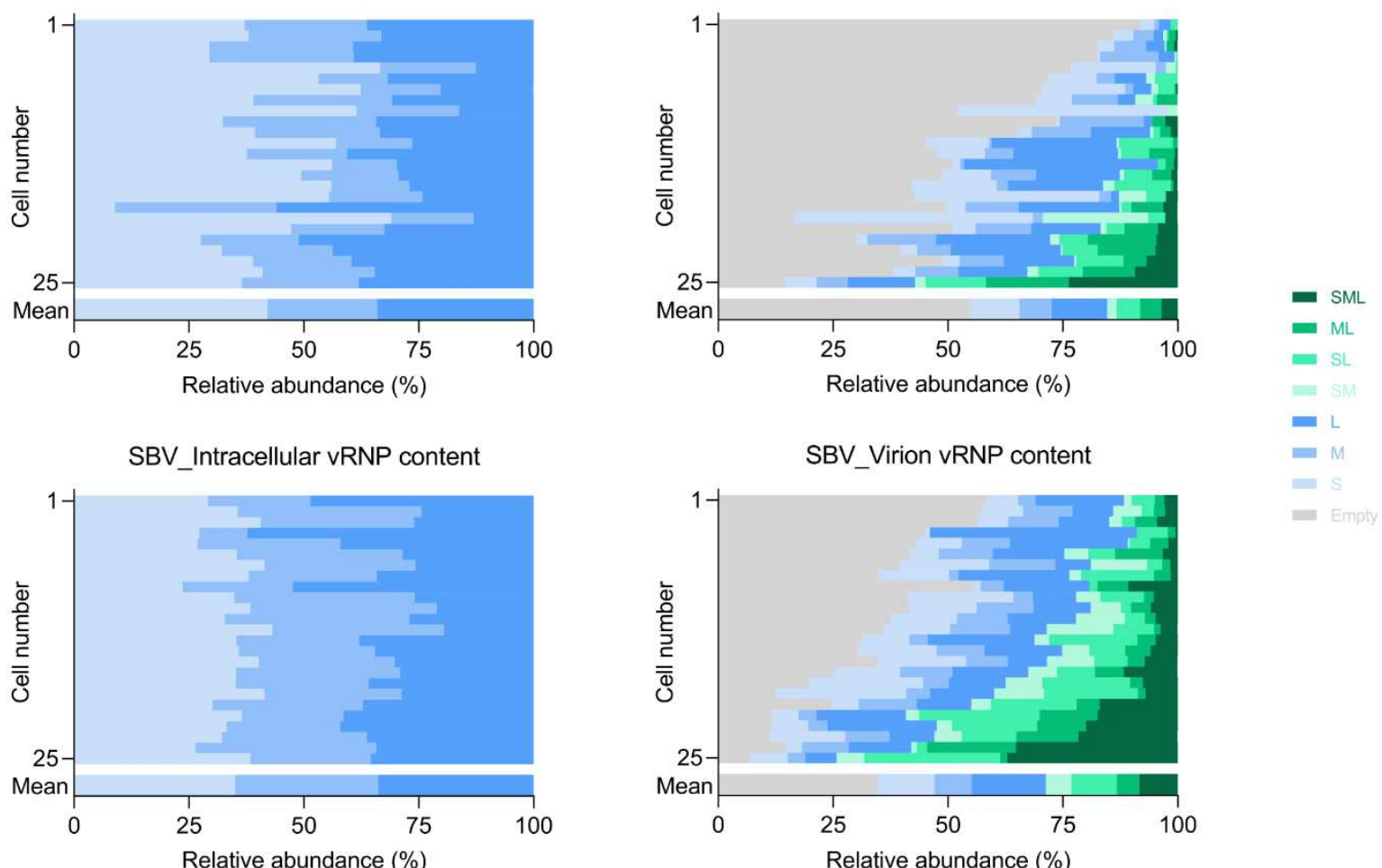

b

SBV_Intracellular vRNP content
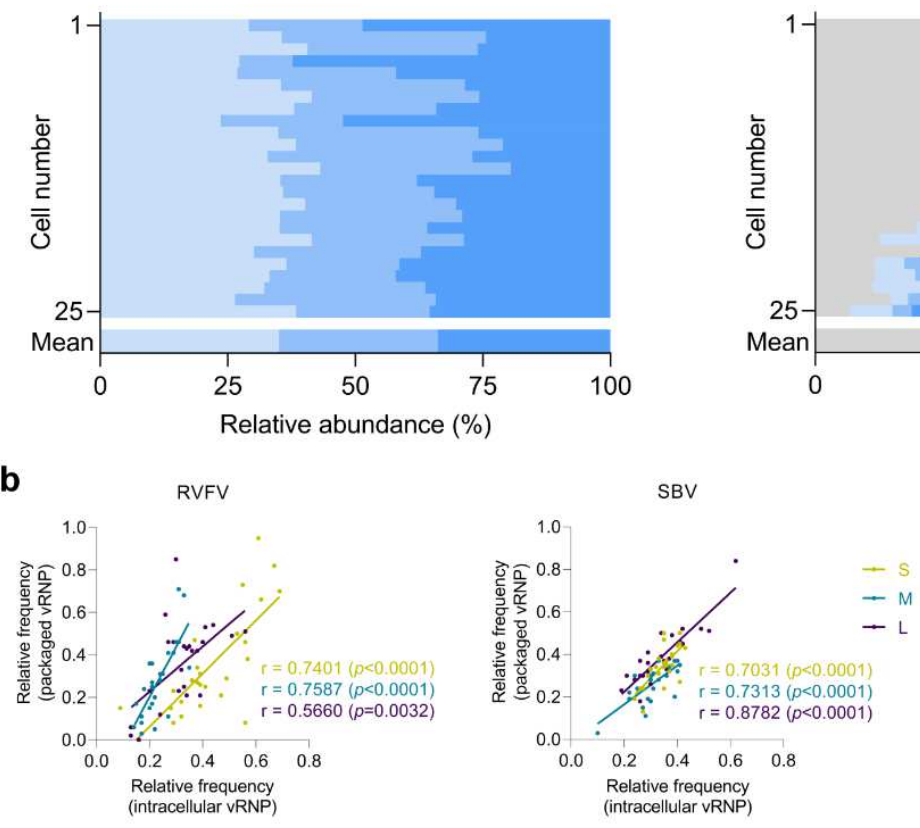

C
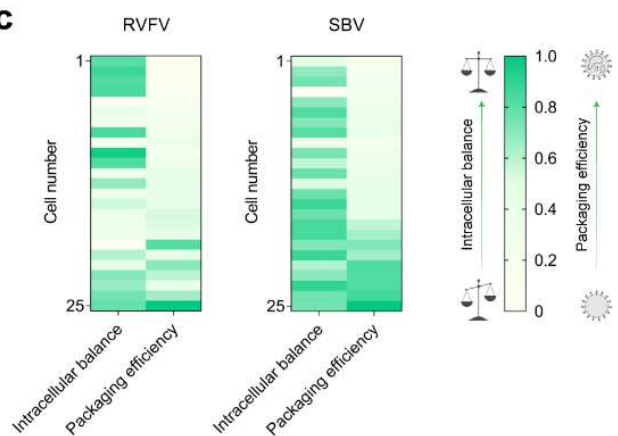

d
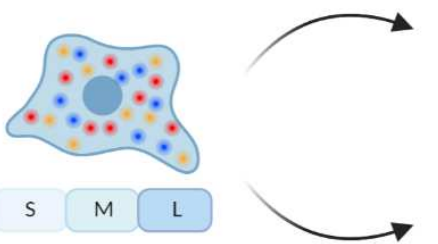

Balanced intracellular VRNP content
Relatively efficient packaging
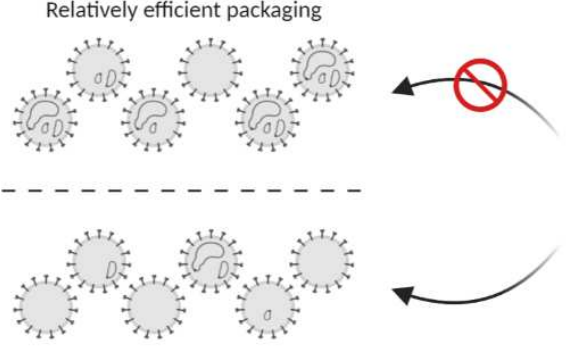

Inefficient packaging

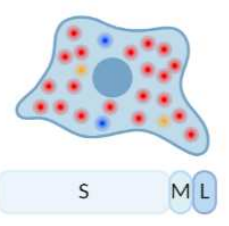

Imbalanced intracellular VRNP content

Fig. 4 VRNP composition of the cytoplasm of bunyavirus infected mammalian (Vero E6) cells and their progeny virions at a single-cell level. RVFV and SBV infected cells were analyzed with a single-molecule vRNA FISHimmunofluorescence method as described in Fig. 3. a Quantification of RVFV and SBV S, M and L VRNPS in the cytoplasm of infected cells (left panels) and in progeny virions (right panels). Data are expressed as the relative intracellular abundance of each VRNP and as the relative abundance of each of the eight different potential compositions of virions. Graphs show the composition results of single cells ( $n=25$ cells; more than 5,000 RVFV virions and more than 4,500 SBV virions) and means. Cell numbers in the left and right panels correspond. 
655 frequency of that genome segment being packaged. Pearson's correlation coefficients ( $r$ ) and $p$ values are shown 656 for each genome segment. c Relationship between the intracellular content of vRNPs and the packaging efficiency 657 of individual cells. A generic system to score the intracellular balance and the packaging efficiency was created. 658 A frequency of 0.33 for each genome segment was considered as theoretically balanced. The balance score was 659 calculated as the summatory of the absolute deviations from the theoretical frequency, normalized from 0 to 1 , 660 assigning the least balanced composition of the data set a score of 0 . The packaging efficiency score was calculated 661 taking into account the frequency of empty, incomplete and complete virus particles, normalized from 0 to 1 , 662 assigning the most efficient packaging value of the data set a score of 1 . Scores are color coded from light green 663 (lowest) to dark green (highest). d Proposed model on the efficiency of genome packaging based on the 664 intracellular VRNP content. A balanced VRNP content in the cytoplasm seems to be a pre-requisite for relatively 665 efficient genome packaging. 


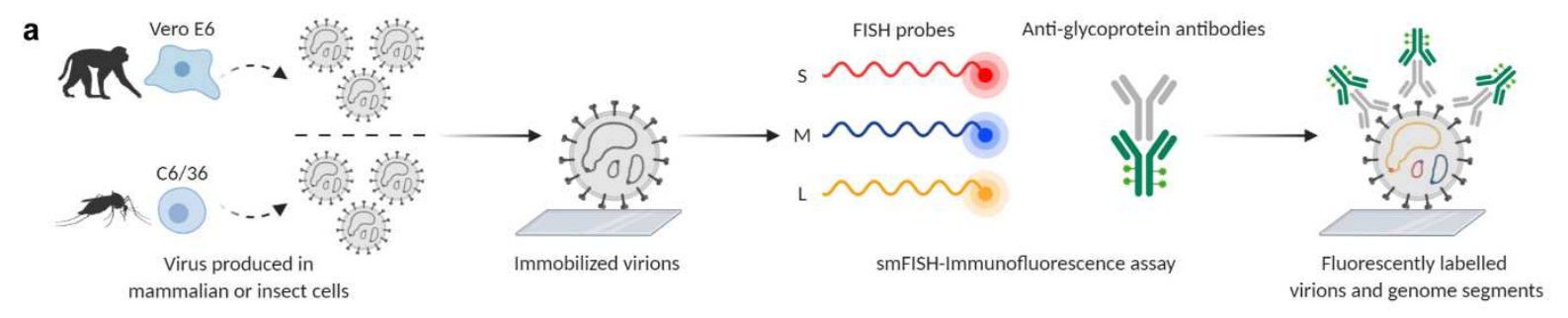

b
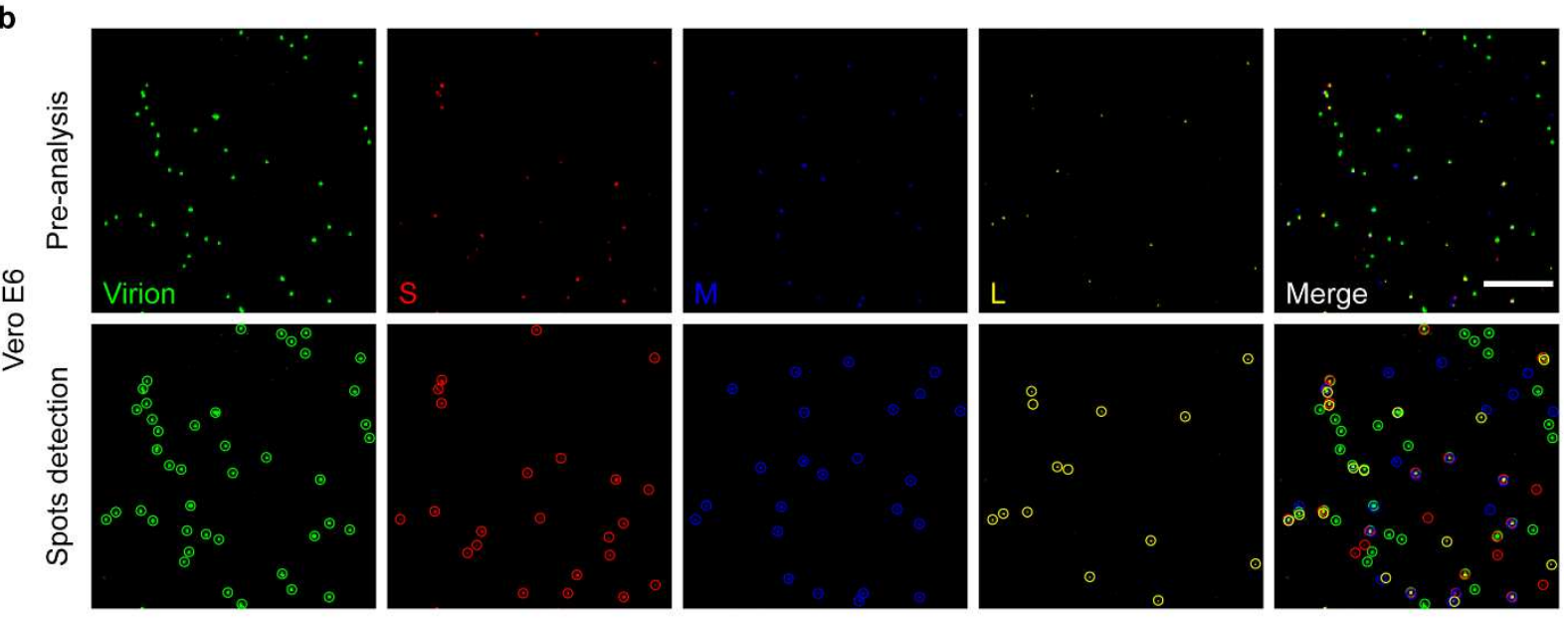

C

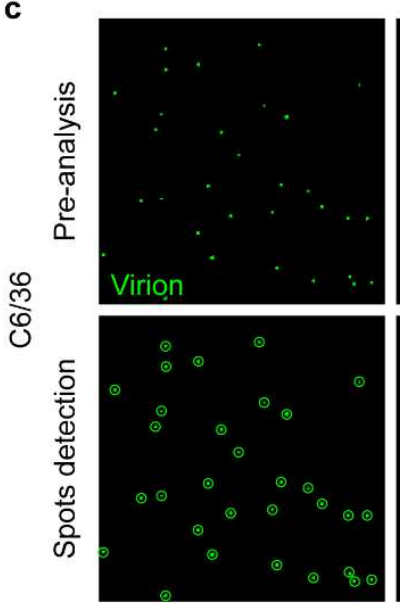

d

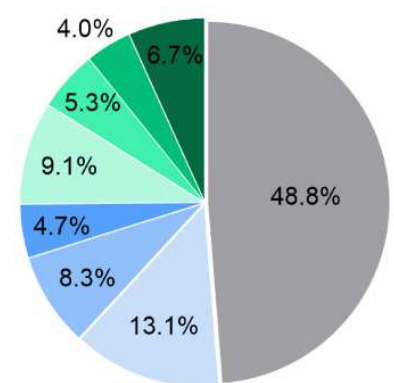

$\mathrm{n}=2981$ virions
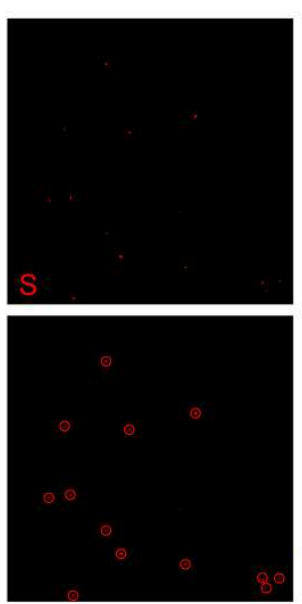

C6/36
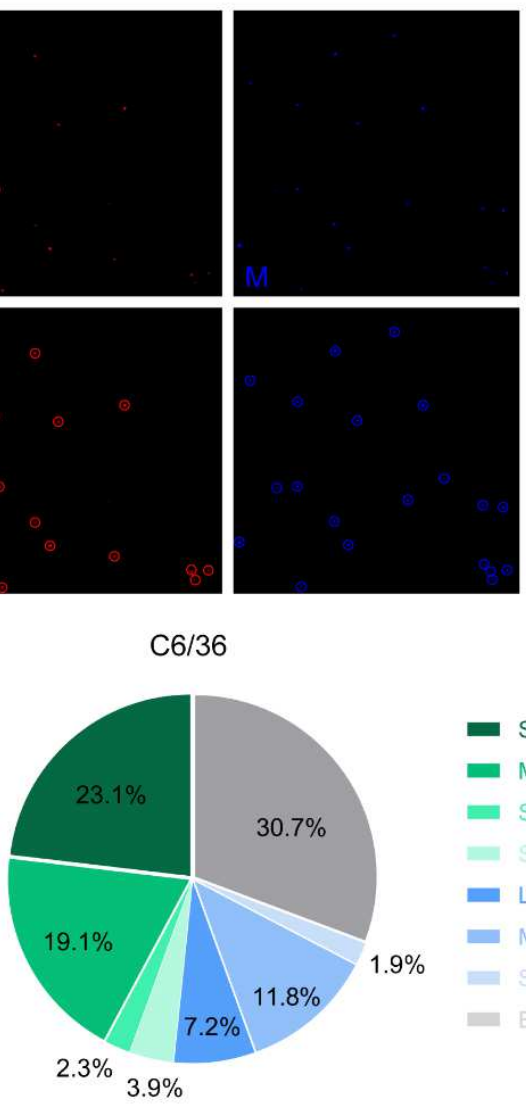

$\mathrm{n}=3040$ virions
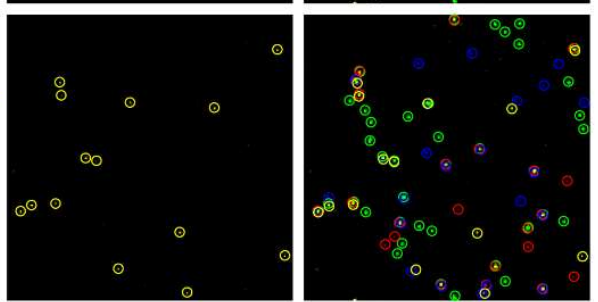

Fig. 5 Genome segment composition of immobilized RVFV virions produced in mammalian and insect cells.

a Schematic representation of the experimental setup. RVFV virions produced in Vero E6 cells or C6/36 cells were 
671 Fluor Red 610, Quasar 670 and Quasar 570, respectively. Progeny RVFV particles (green) were detected with

672 antibody 4-D4 ${ }^{48}$ targeting the Gn glycoprotein in combination with Alexa Fluor 488-conjugated secondary

673 antibodies. Individual spots, each representing either a single vRNP or a virus particle were detected, counted and

674 assessed for co-localization in ImageJ with the plugin ComDet. b, c Visualization of RVFV virions produced in

675 Vero E6 cells (b) or C6/36 cells (c) (top rows). Merge images show the overlay of the four individual channels.

676 Colored circles (bottom rows) display the spots detected on each channel and their co-localization in the merge

677 image. Due to a higher fluorescence intensity of the green channel compared to the other channels, spots

678 co-localizing with the glycoprotein may sometimes appear masked and not entirely evident in merged images.

679 Scale bars, $5 \mu \mathrm{m}$. d Relative abundance of the eight possible genome compositions of the virions produced in

680 Vero E6 cells (left) or C6/36 cells (right). e Abundance of each genome segment incorporated into a virion relative

681 to the total genome segment packaging events. 
a
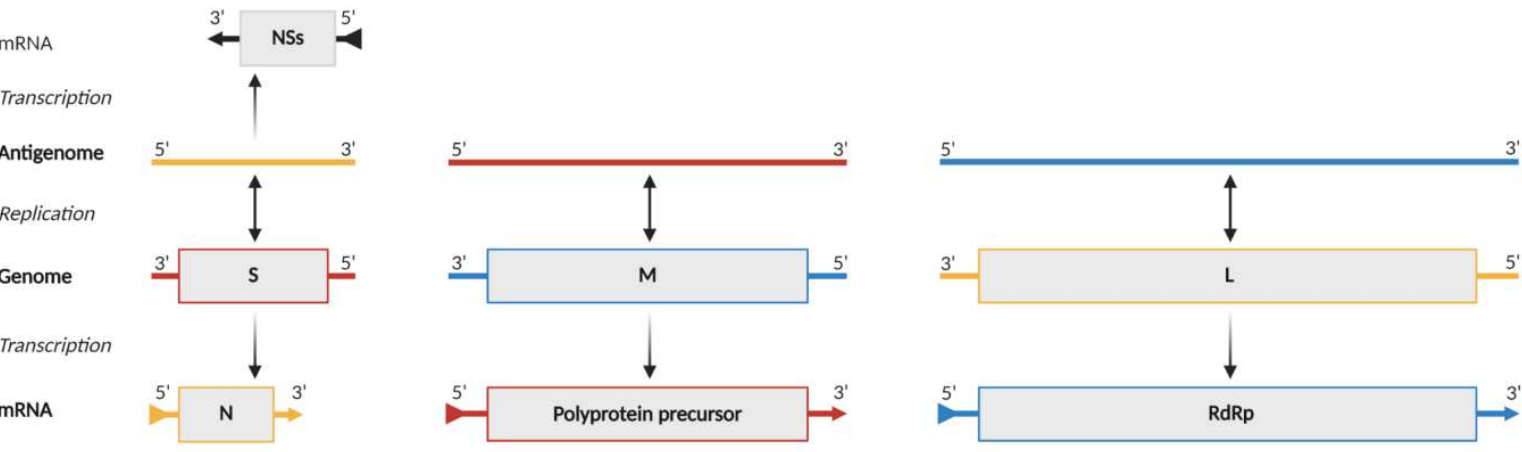

b
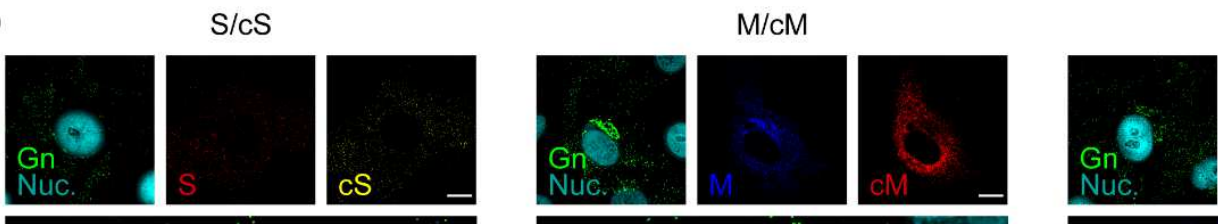

L/CL
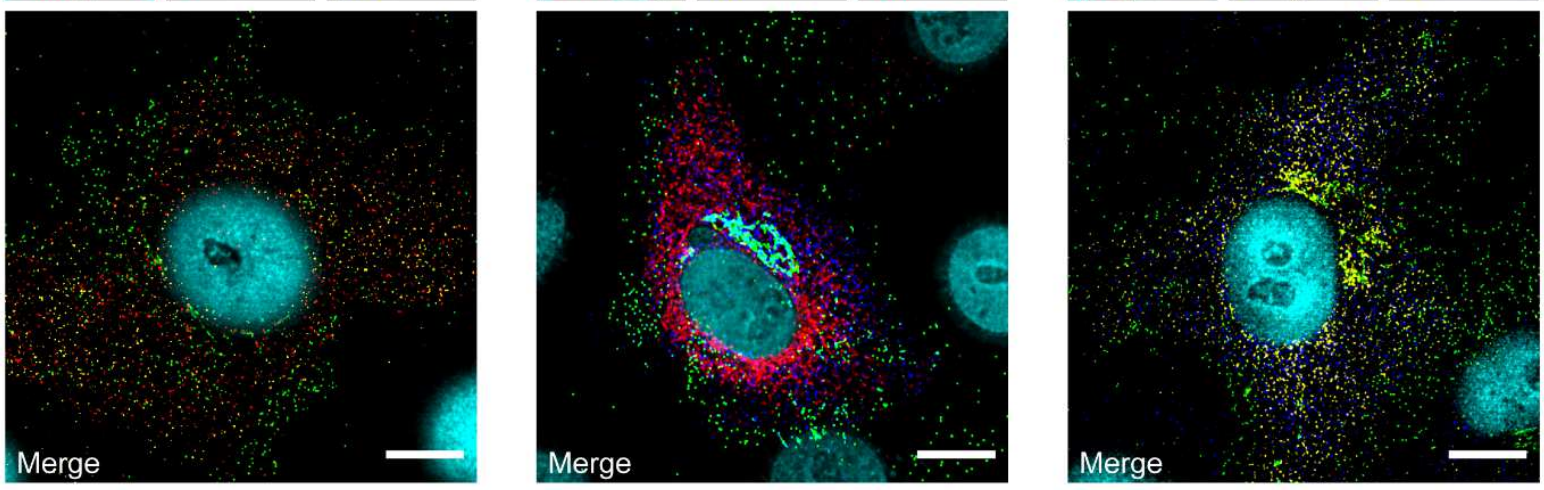

c

S/cS ratio $\approx 4: 1$

$\mathrm{M} / \mathrm{cM}$ ratio $\approx 9: 1$
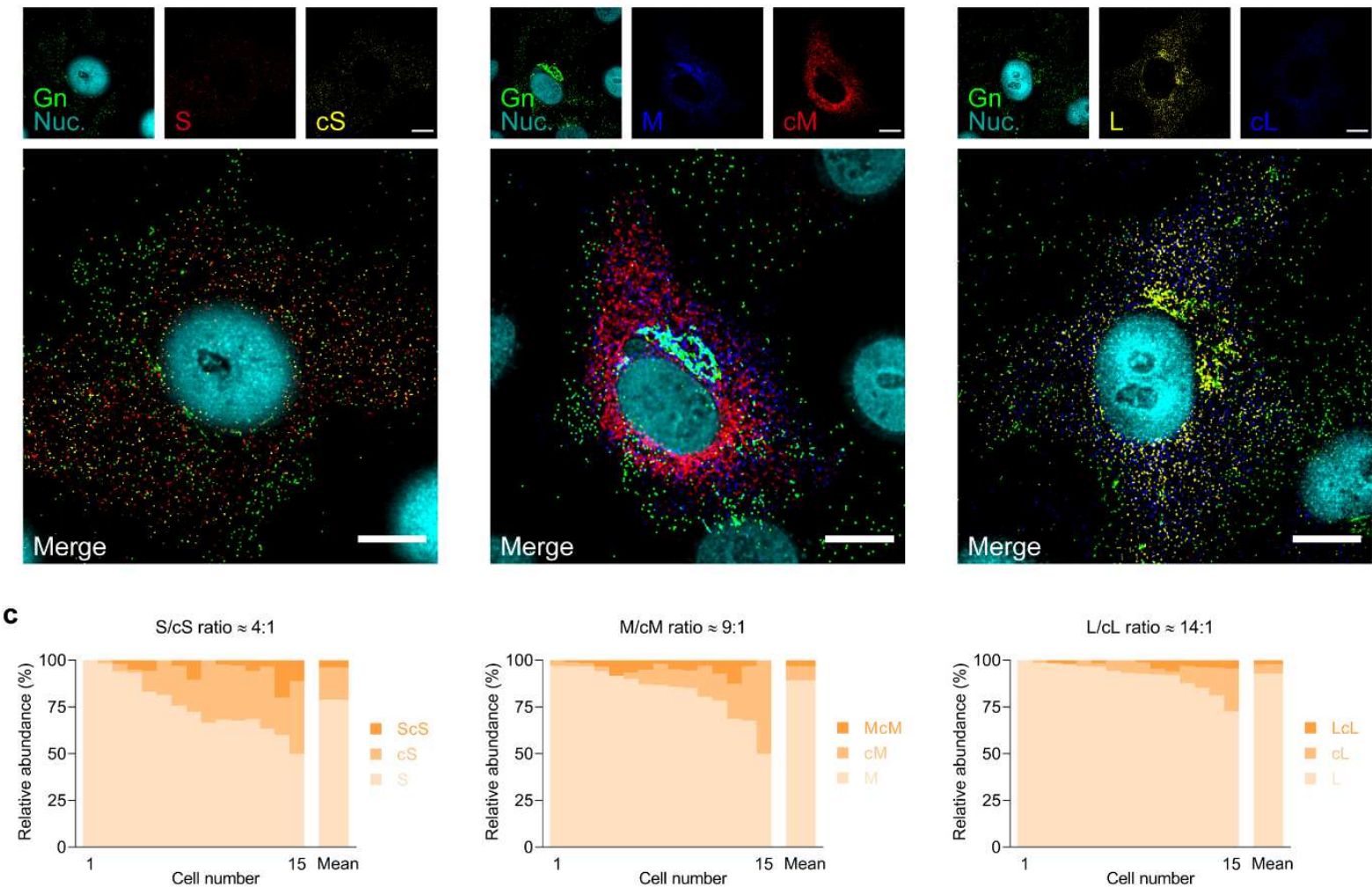

Fig. 6 Single-molecule RNA FISH-immunofluorescence on VRNA-cRNA pairs of RVFV infected mammalian cells. a Schematic representation of the replication and transcription of RVFV genome segments. Here, we refer to viral genome replication intermediates (antigenomes) and mRNA transcripts as cRNAs. RVFV S segment uses an ambisense coding strategy to generate mRNAs from both the genomic-sense and antigenomic-sense RNAs. b Visualization of VRNPs, cRNAs and progeny virions of RVFV infected cells. Vero E6 cells were infected with RVFV ( $\mathrm{MOI}$ 0.75-1.00) and cells were fixed at 8-10 h post-infection. Samples were hybridized against paired targets (i.e. S-cS segments, M-cM segments and L-cL segments). The S segment ( $\mathrm{N}$ gene; red), M segment (polyprotein gene; blue) and L segment (RdRp gene; yellow) were hybridized using probe sets labelled with CAL Fluor Red 610, Quasar 670 and Quasar 570, respectively. The cS segment (N gene; yellow), cM segment (polyprotein gene; red) and CL segment (RdRp gene; blue) were hybridized using probe sets labelled with Quasar 570, CAL Fluor Red 610 and Quasar 670, respectively. Progeny RVFV particles (green) were detected with antibody 4-D4 ${ }^{48}$ targeting the Gn glycoprotein in combination with Alexa Fluor 488-conjugated secondary antibodies. Cell nuclei (cyan) were visualized with DAPI. Individual spots, each representing either a VRNP, a cRNA or a virus particle were detected, 
counted and assessed for co-localization in ImageJ with the plugin ComDet. Main images are merged maximum intensity projections of four channels (individual channels shown on top). Due to a higher fluorescence intensity

698 of the green channel compared to the other channels, spots co-localizing with the glycoprotein may sometimes appear masked and not entirely evident in merged images. Scale bars, $10 \mu \mathrm{m}$. c Quantification of the S, M, L vRNPS and their corresponding cRNAs in RVFV progeny virions. Genome compositions of the virions are expressed as their abundance relative to the amount of virions in which at least one vRNP or cRNA was detected. Graphs show the composition results of virions released by single cells ( $n=15$ cells per combination; more than 3,900 virions per combination) and means. vRNA/cRNA ratios are indicated. cS, complementary S segment; cM, complementary M segment; $\mathrm{CL}$, complementary $L$ segment. 
Figures

a
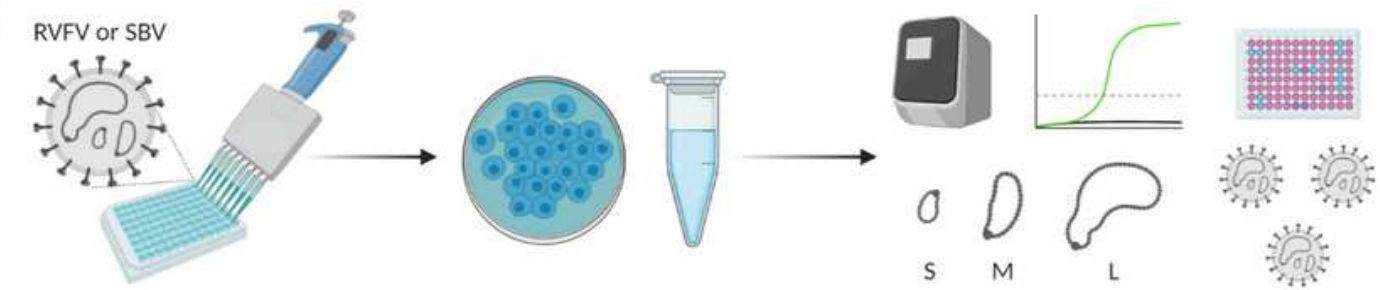

Infection of mammalian (Vero E6)

Collection of cell lysates and supernatants

Quantification of genome segments and infectious virus or insect ( $66 / 36$ or $\mathrm{KC})$ cells

d

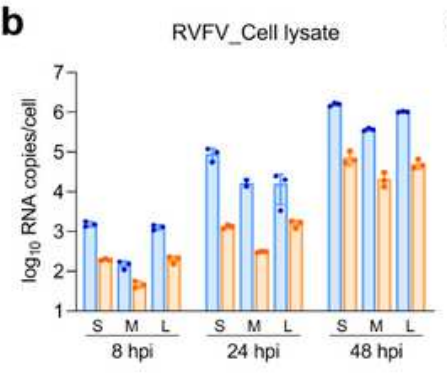

C

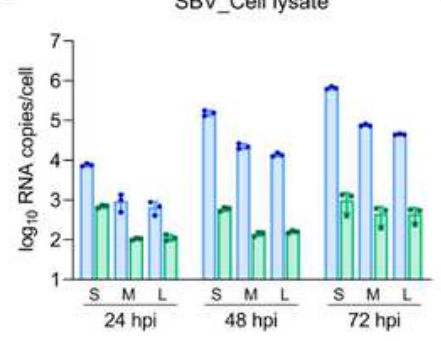

RVFV Supernatant

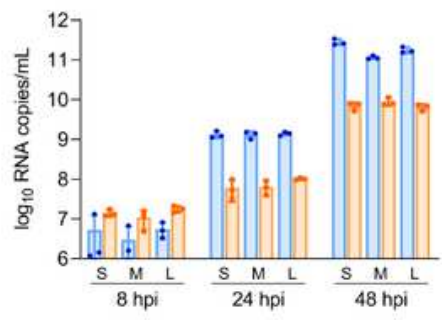

SBV Supernatant

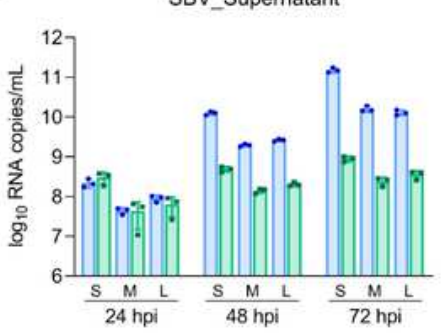

f

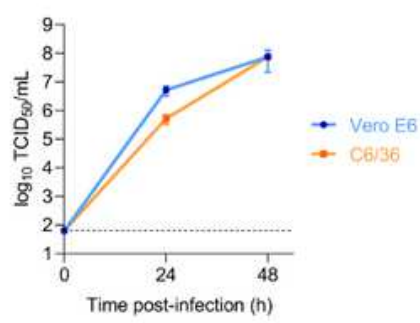

g

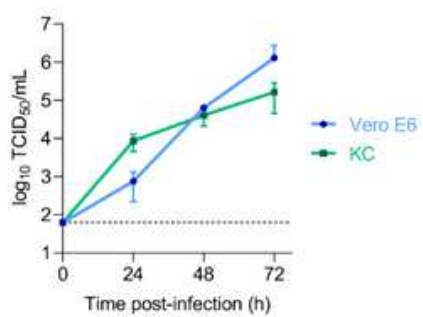

h

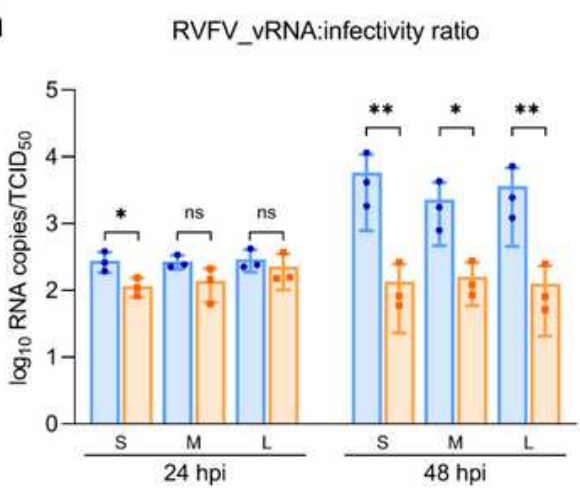

i

SBV_VRNA:infectivity ratio
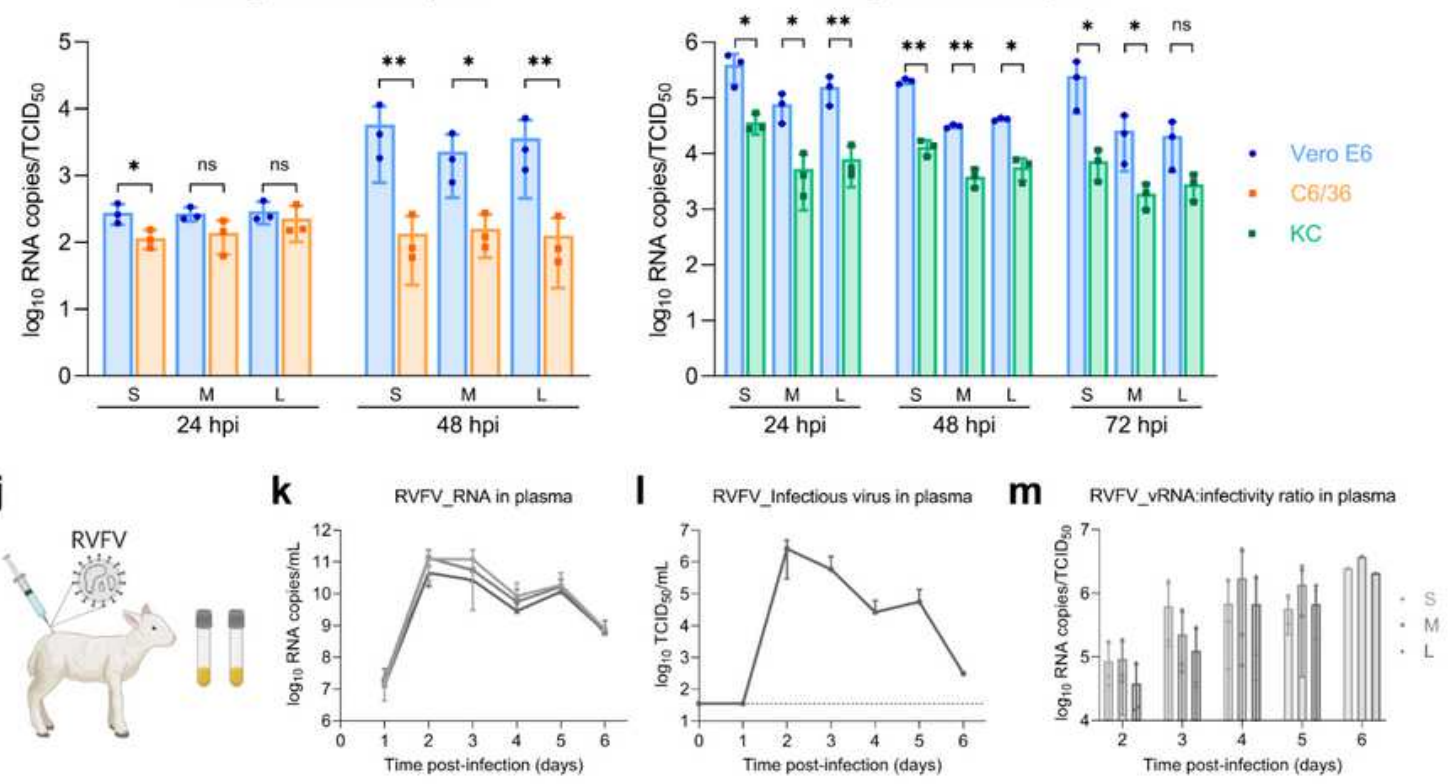

Figure 1

Viral RNA:infectivity ratios in mammalian and insect hosts. a Schematic representation of the in vitro 584 experimental setup. Mammalian (Vero E6) and insect (C6/36 and KC) cells were infected with RVFV or SBV (MOI 0.01). Cell lysates and supernatants were collected at defined time points. Viral RNA was 
quantified withgenome segment-specific RT-qPCRs and virus titers were determined by endpoint titration. b-e In vitro replication kinetics of RVFV and SBV. Bars show means with SD. Dots represent biological replicates ( $n=3$ samplest). + Bar of RVFV cell lysate M segment at $24 \mathrm{~h}$ post-infection shows mean of two samples. $\mathrm{f}, \mathrm{g}$ RVFV and SBV infectious titers in cell culture supernatants. Titers correspond to the same supernatant samples analyzed in $d$, e. Graphs show means with SD of $n=3$ biological replicates. The dashed line indicates the limit of detection (101.80 TCID50/mL). h, i RVFV and SBV vRNA:infectivity ratios calculated as viral genome copies per infectious unit in cell culture supernatants. Bars show means with SD. Dots represent individual ratios $(n=3)$. j Schematic representation of the animal samples from another study obtained for analysis. Lambs were experimentally infected via intravenous route with RVFV and plasma samples were collected daily28. $k$ In vivo replication kinetics of RVFV. Graph shows means with SD of plasma samples $(n=3 \ddagger)$ analyzed by RT-qPCR. I RVFV infectious titers in plasma as determined with a virus isolation assay28. Graph shows means with SD of plasma samples $(n=3 \ddagger)$. The dashed line indicates the limit of detection (101.55 TCID50/mL). m RVFV in vivo vRNA:infectivity ratios calculated as viral genome copies per infectious unit in plasma. Bars show means with SD. Dots represent individual ratios $(n=3 \ddagger)$. $\ddagger$ At early ( 1 day) and late $(5-6$ days) times post-infection, genome copies and infectious titers of some samples were below the limits of detection. In those cases, the reported values represent the mean of two samples or a single sample. Statistical analysis of vRNA:infectivity ratios was performed using an unpaired two-tailed Student's $t$ test with Welch's correction (not assuming equal variances). ${ }^{*} p<0.05 ;{ }^{*} p<0.01 ; n s$, not significant $(p \geq 0.05)$. 
a

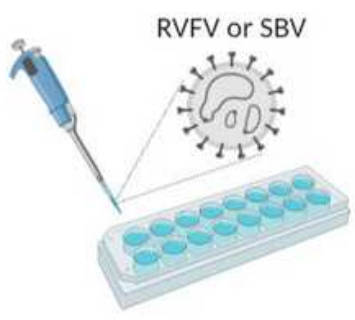

Infection of Vero E6 cells

b

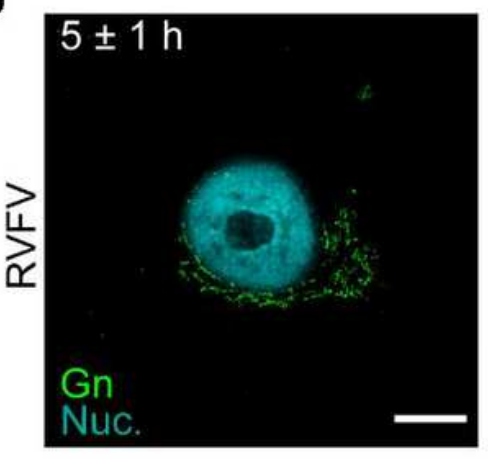

C

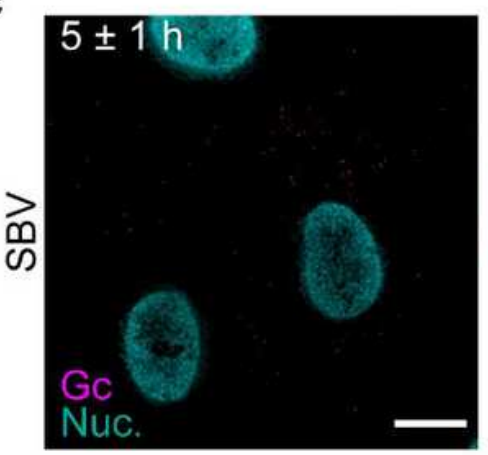

f

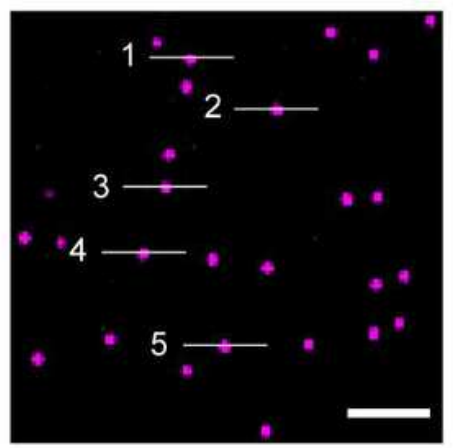

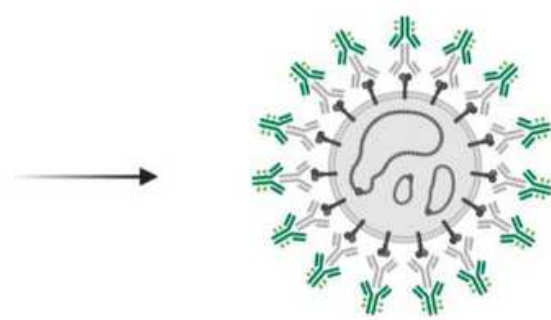

Immunofluorescence detection of Gn (RVFV) or Gc (SBV)

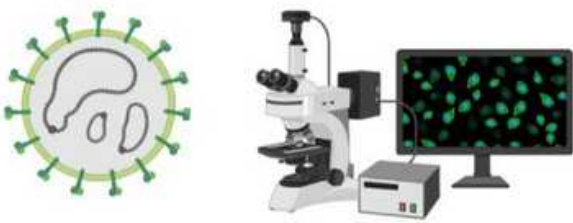

Visualization of progeny virions in infected cells

d
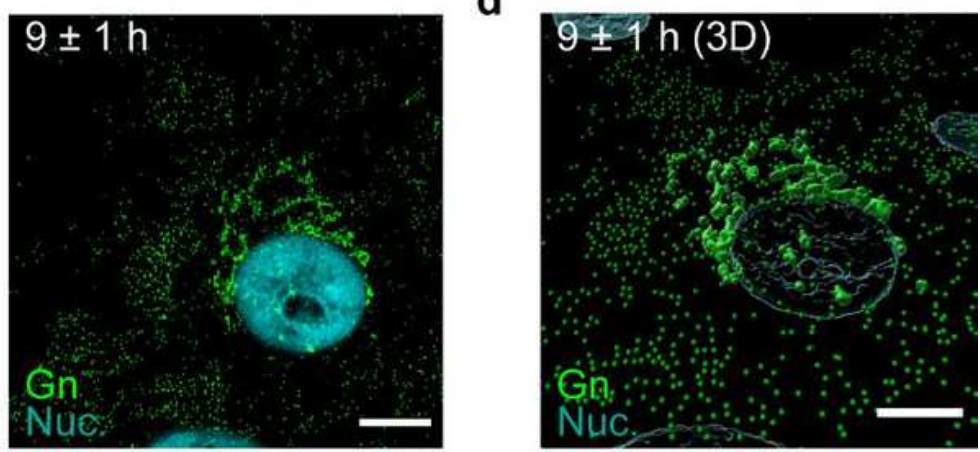

e

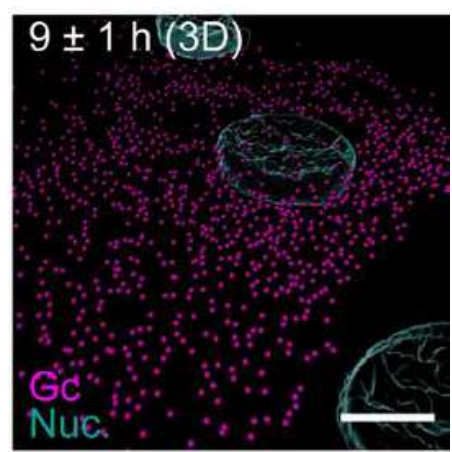

g

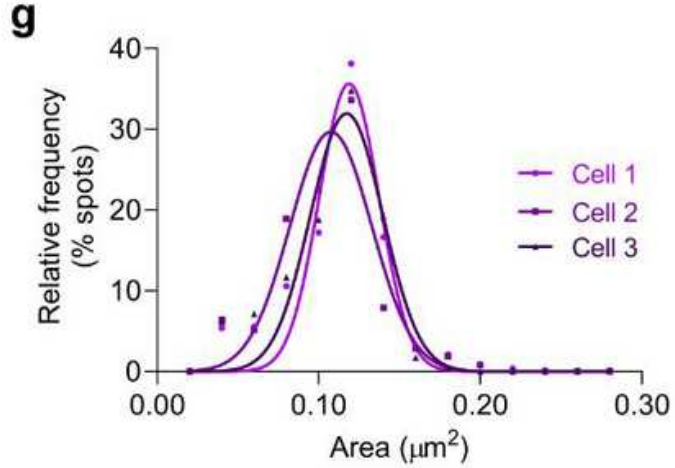

Figure 2

Immunofluorescence detection of newly formed bunyavirus progeny virions at single-particle resolution. a Schematic representation of the experimental setup. Vero E6 cells were infected with RVFV (MOI 1) or SBV (MOI 0.33) and cells were fixed at defined time points. Progeny virions were detected by immunofluorescence. b, c Release kinetics of RVFV particles (green) (b) and SBV particles (magenta) (c). RVFV virions were detected with antibody 4-D448 targeting the Gn glycoprotein in combination with Alexa Fluor 488-conjugated secondary antibodies. SBV virions were detected with serum from an immunized 
rabbit52 targeting the Gc glycoprotein in combination with FITC-conjugated secondary antibodies. Cell nuclei (cyan) were visualized with DAPI. RVFV Gn accumulates in a perinuclear region, the site of virion assembly. $\mathrm{d}$, e Three-dimensional representations showing the spatial distribution of virions at the $9 \pm 1 \mathrm{~h}$ time point created with Imaris using the Surfaces and Spots modes. $f$ Magnification of a region of interest (indicated as a dashed box in the second panel of c) and fluorescence intensity plot of the indicated spots. Dots represent data points and lines show Gaussian curves fitting the data. The unimodal distribution of fluorescence intensities along the lines crossing the spots is characteristic of single particles. g Histogram of the area of the spots detected in images of SBV infected cells $(n=3$ cells; more than 500 spots per image). Dots represent data points and lines show Gaussian curves fitting the data. The unimodal distribution denotes reproducible measurements of single spots within and between images. Images are merged maximum intensity projections of two channels. Scale bars, $10 \mu \mathrm{m}(\mathrm{b}-\mathrm{e}), 2$ $\mu \mathrm{m}(\mathrm{f})$. 


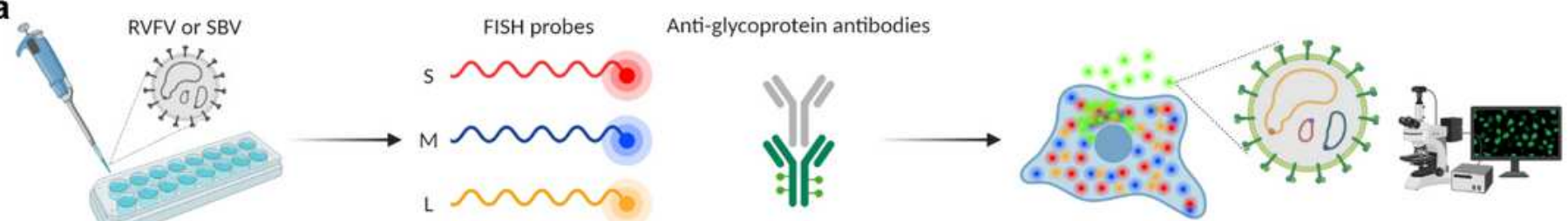

b

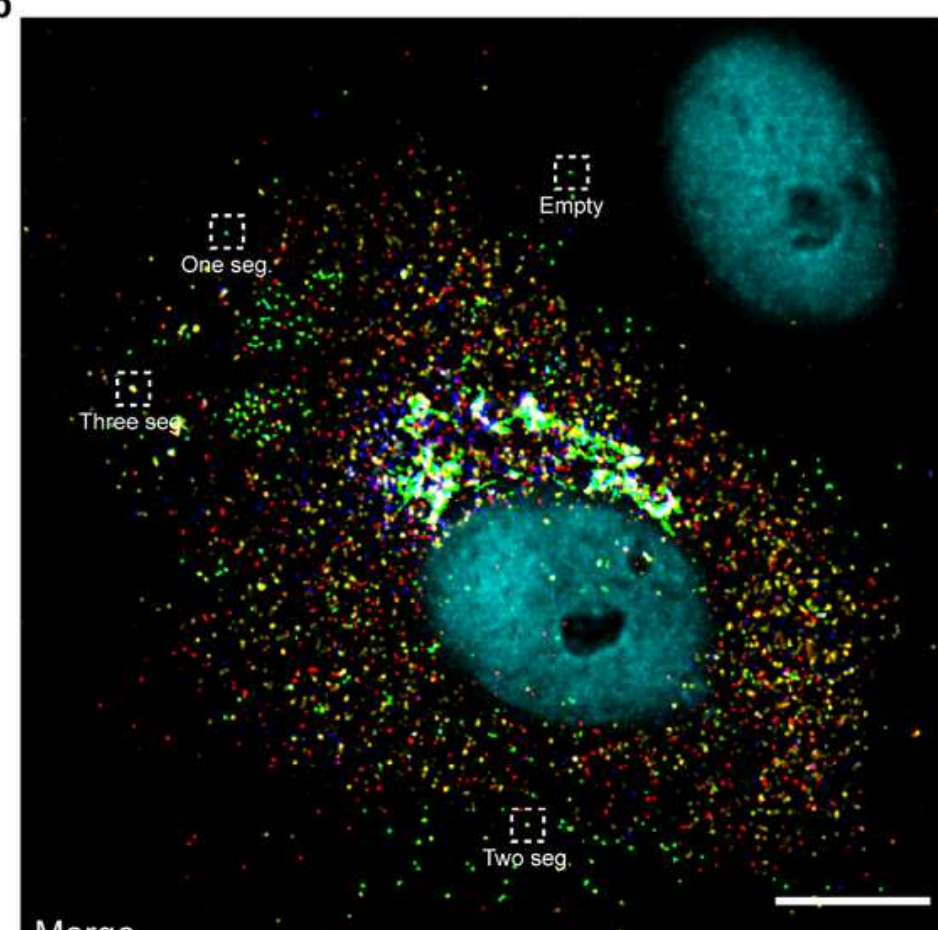

C
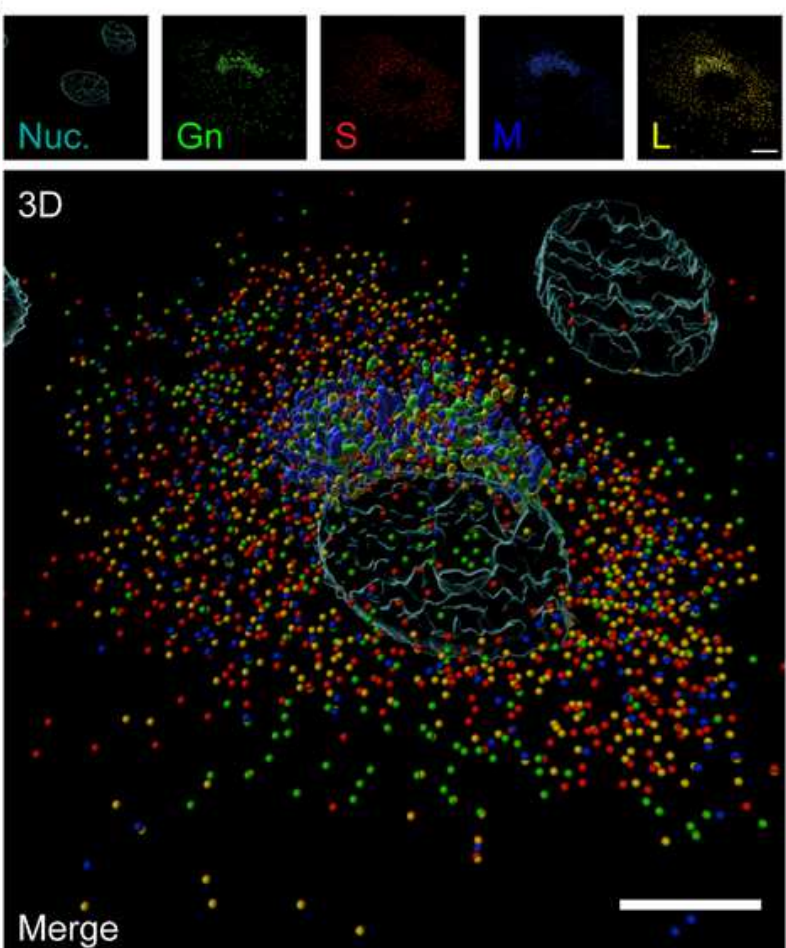

d
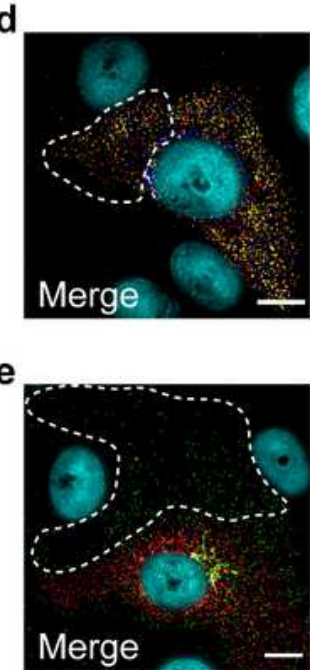

f
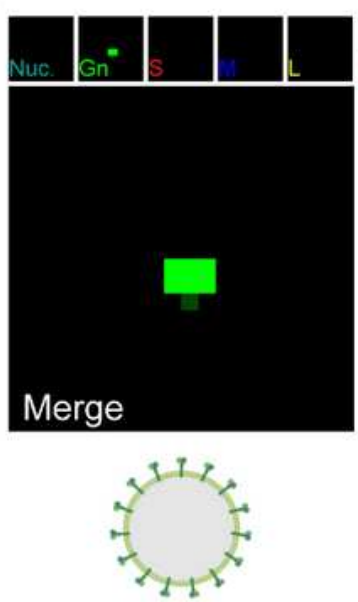

Empty particle

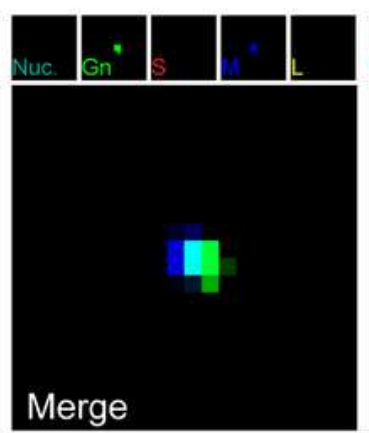

Merge
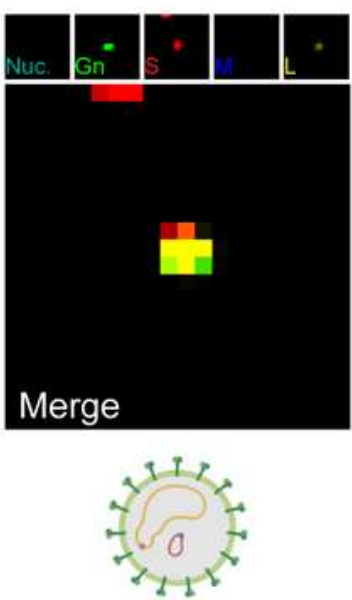

Two segments (SL)
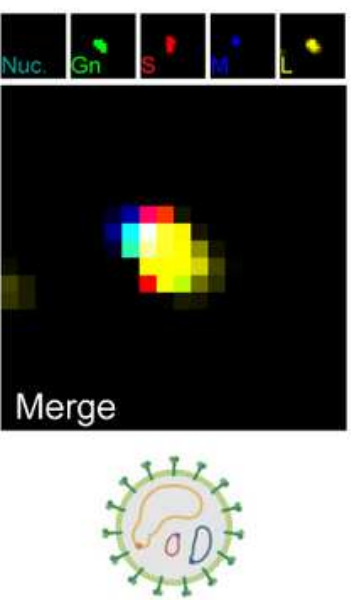

Three segments (SML)

\section{Figure 3}

Single-molecule vRNA FISH-immunofluorescence of bunyavirus infected mammalian cells. a Schematic representation of the experimental setup. Vero E6 cells were infected with RVFV (MOI 0.50-0.75) or SBV ( $\mathrm{MOI} 0.33$ ) and cells were fixed at $8 \mathrm{~h}$ post-infection. The $\mathrm{S}$ segment ( $\mathrm{N}$ gene; red), $\mathrm{M}$ segment (polyprotein gene; blue) and L segment (RdRp gene; yellow) were hybridized using probe sets labelled with CAL Fluor Red 610, Quasar 670 and Quasar 570, respectively. Progeny RVFV particles (green) were 
detected with antibody 4-D448 targeting the Gn glycoprotein in combination with Alexa Fluor 488conjugated secondary antibodies. Progeny SBV particles (Supplementary Figs. 3b, 4) were detected with serum from an immunized rabbit52 targeting the Gc glycoprotein in combination with FITC-conjugated secondary antibodies. Cell nuclei (cyan) were visualized with DAPI. Individual spots, each representing either a single VRNP or a virus particle were detected, counted and assessed for co-localization in ImageJ with the plugin ComDet. b Visualization of vRNPs and progeny virions of a RVFV infected cell. The dashed boxes highlight individual virus particles subjected to co-localization analysis for example purposes. The number of RVFV genome segments in each highlighted particle is indicated. c Threedimensional representation showing the spatial distribution of vRNPs and virions of the image displayed in $b$ created with Imaris using the Surfaces and Spots modes. Accumulation of vRNPs and co-localization to the same perinuclear region as Gn show active vRNP recruitment to the site of virion assembly. Colocalization of vRNPs and virions is depicted by merged spheres. d, e RVFV infected cells. The dashed contours represent example regions of interest selected for quantification of cytoplasmic vRNPs (d) and determining the genome composition of extracellular virions through co-localization analysis (e) (Supplementary Fig. 3a). Example regions of interest selected for analysis of SBV infected cells are shown in Supplementary Fig. 3b. $\mathrm{f}$ Magnification of regions of interest indicated by dashed boxes in $\mathrm{b}$. The genome composition of each virion can be deduced from the spots detected on each individual channel. Images are merged maximum intensity projections of four (d) or five (b, c, e, f) channels. Due to a higher fluorescence intensity of the green channel compared to the other channels, spots co-localizing with the glycoprotein may sometimes appear masked and not entirely evident in merged images. Scale bars, $10 \mu \mathrm{m}$. 
a

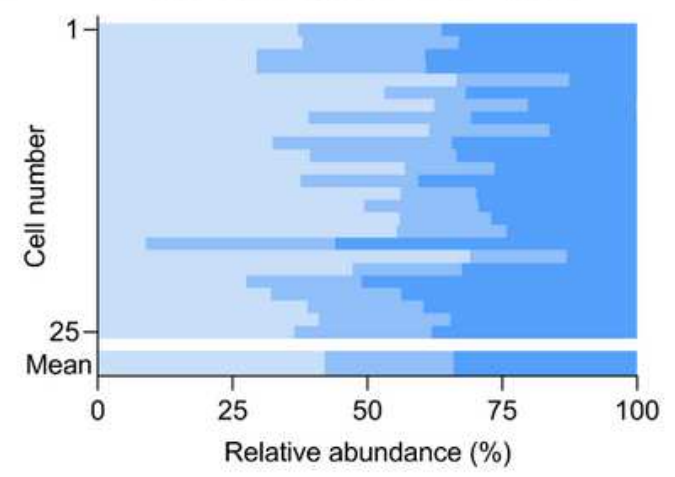

SBV_Intracellular vRNP content

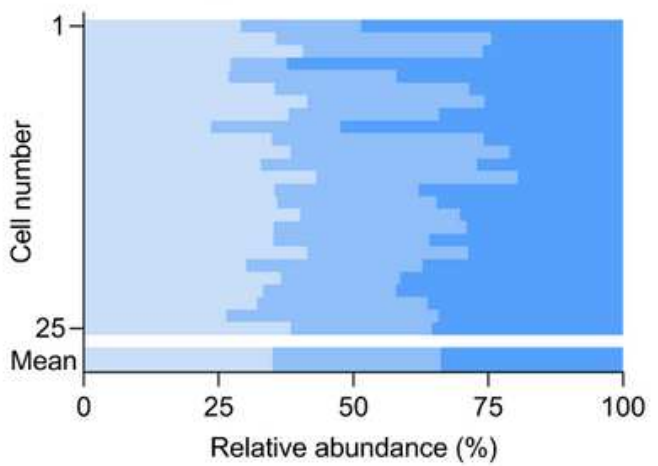

b
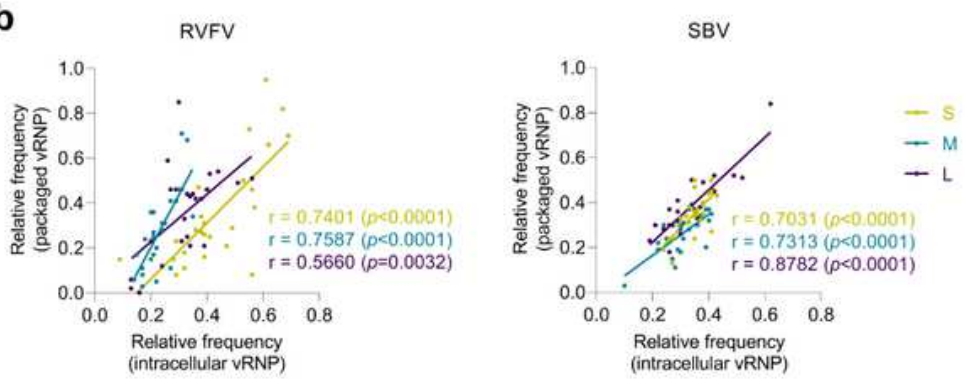

d

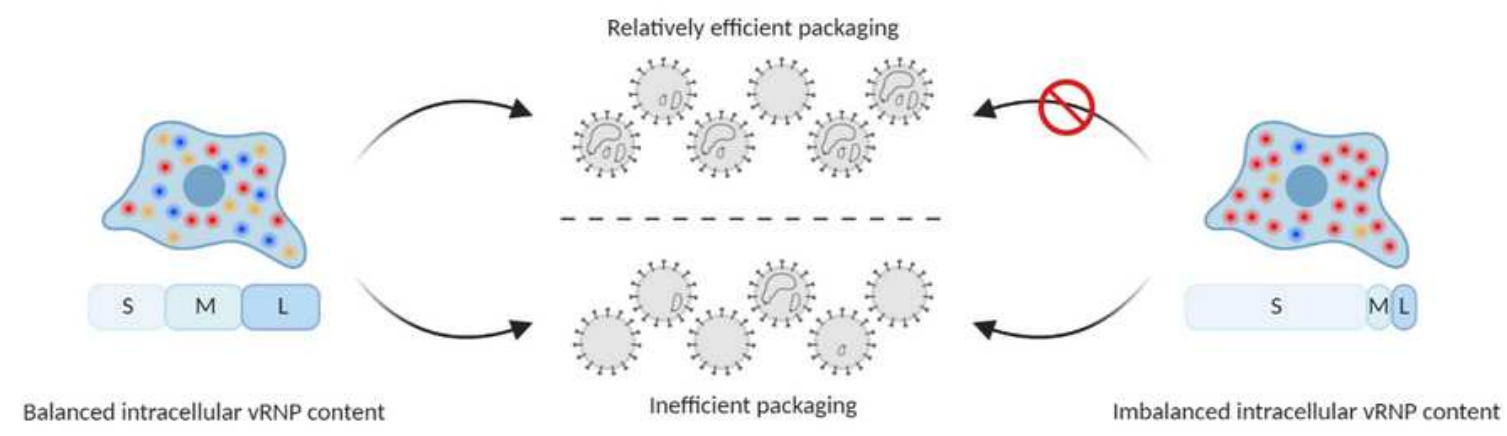

\section{Figure 4}

vRNP composition of the cytoplasm of bunyavirus infected mammalian (Vero E6) cells and their progeny virions at a single-cell level. RVFV and SBV infected cells were analyzed with a single-molecule vRNA FISH- immunofluorescence method as described in Fig. 3. a Quantification of RVFV and SBV S, M and L vRNPs in the cytoplasm of infected cells (left panels) and in progeny virions (right panels). Data are expressed as the relative intracellular abundance of each VRNP and as the relative abundance of each of 
the eight different potential compositions of virions. Graphs show the composition results of single cells ( $n=25$ cells; more than 5,000 RVFV virions and more than 4,500 SBV virions) and means. Cell numbers in the left and right panels correspond. b Correlation analysis between the relative intracellular frequency of a specific genome segment and the relative frequency of that genome segment being packaged. Pearson's correlation coefficients $(r)$ and $p$ values are shown for each genome segment. c Relationship between the intracellular content of vRNPs and the packaging efficiency of individual cells. A generic system to score the intracellular balance and the packaging efficiency was created. A frequency of 0.33 for each genome segment was considered as theoretically balanced. The balance score was calculated as the summatory of the absolute deviations from the theoretical frequency, normalized from 0 to 1 , assigning the least balanced composition of the data set a score of 0 . The packaging efficiency score was calculated taking into account the frequency of empty, incomplete and complete virus particles, normalized from 0 to 1 , assigning the most efficient packaging value of the data set a score of 1 . Scores are color coded from light green (lowest) to dark green (highest). d Proposed model on the efficiency of genome packaging based on the intracellular vRNP content. A balanced vRNP content in the cytoplasm seems to be a pre-requisite for relatively efficient genome packaging. 
a

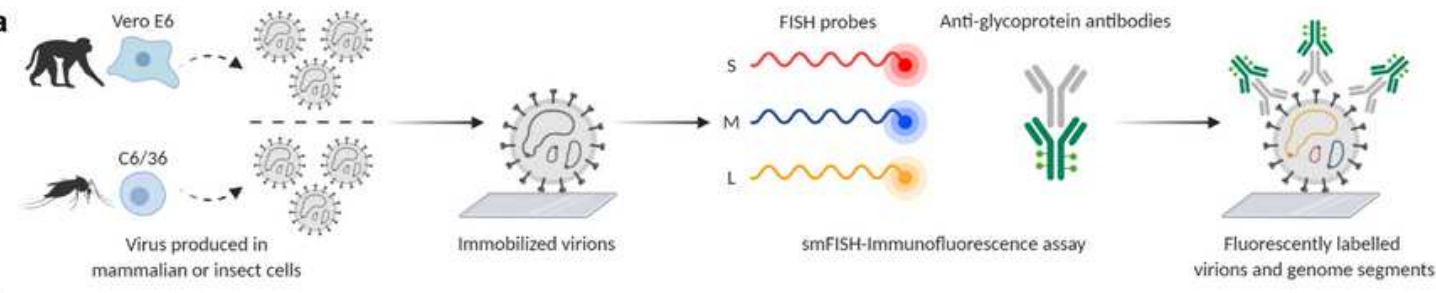

b

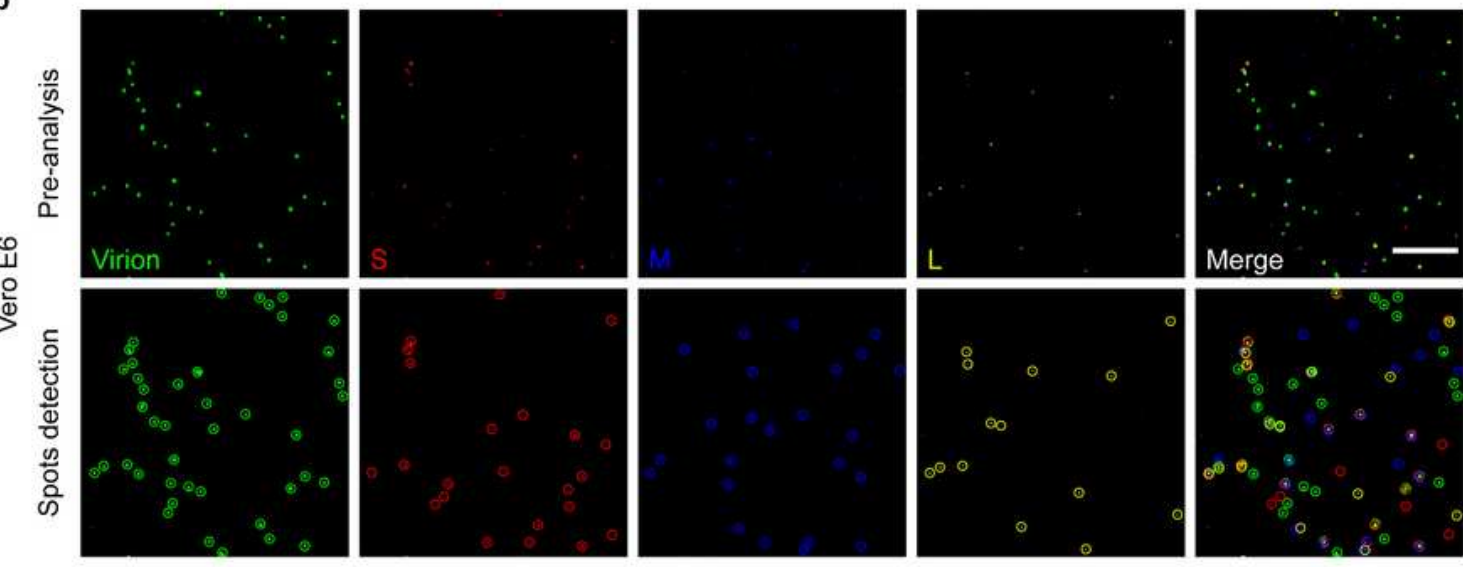

c
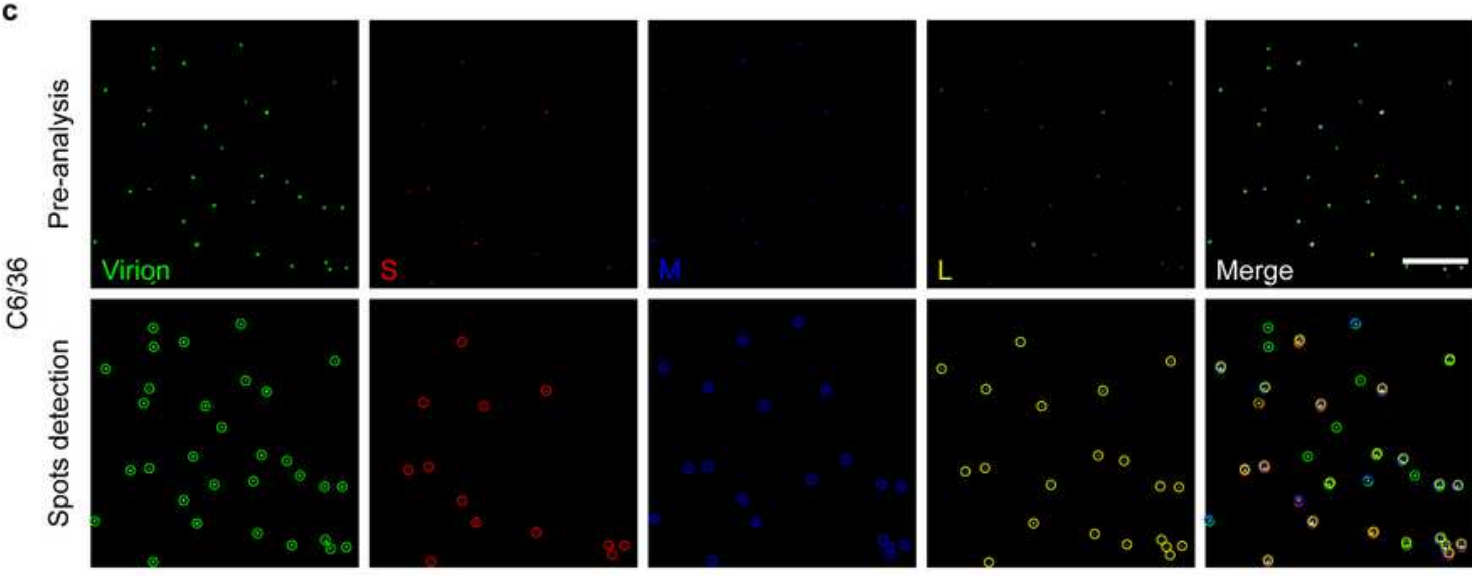

d

Vero E6

$\mathrm{C} 6 / 36$
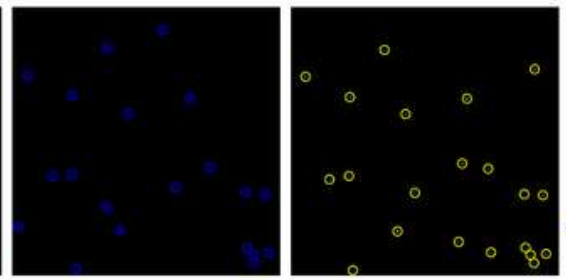

e Segment preference

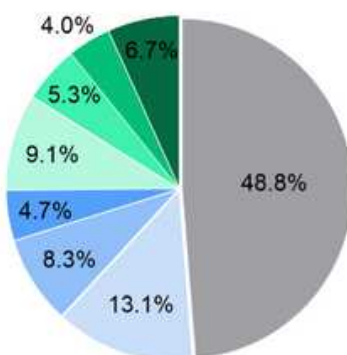

$\mathrm{n}=2981$ virions

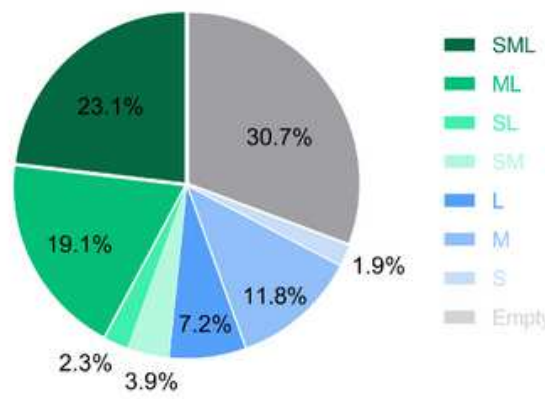

$n=3040$ virions

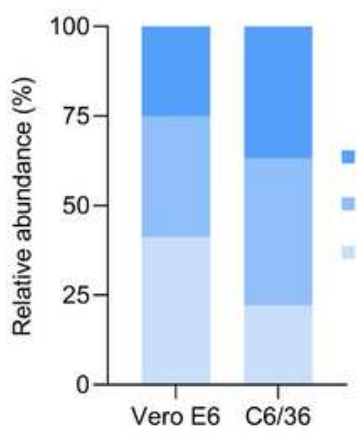

\section{Figure 5}

Genome segment composition of immobilized RVFV virions produced in mammalian and insect cells. a Schematic representation of the experimental setup. RVFV virions produced in Vero E6 cells or C6/36 cells were immobilized on coverglass by incubation for $5 \mathrm{~h}$ at $28^{\circ} \mathrm{C}$. The $\mathrm{S}$ segment ( $\mathrm{N}$ gene; red), $\mathrm{M}$ segment (NSmGn and Gc genes separately; blue) and L segment (RdRp gene; yellow) were hybridized using probe sets labelled with CAL Red 610, Quasar 670 and Quasar 570, respectively. Progeny RVFV 
particles (green) were detected with antibody 4-D448 targeting the Gn glycoprotein in combination with Alexa Fluor 488-conjugated secondary antibodies. Individual spots, each representing either a single vRNP or a virus particle were detected, counted and assessed for co-localization in ImageJ with the plugin ComDet. b, c Visualization of RVFV virions produced in Vero E6 cells (b) or C6/36 cells (c) (top rows). Merge images show the overlay of the four individual channels. Colored circles (bottom rows) display the spots detected on each channel and their co-localization in the merge image. Due to a higher fluorescence intensity of the green channel compared to the other channels, spots co-localizing with the glycoprotein may sometimes appear masked and not entirely evident in merged images. Scale bars, 5 $\mu \mathrm{m}$. $\mathrm{d}$ Relative abundance of the eight possible genome compositions of the virions produced in Vero E6 cells (left) or $\mathrm{C} 6 / 36$ cells (right). e Abundance of each genome segment incorporated into a virion relative to the total genome segment packaging events. 
a $\stackrel{3^{\prime}}{\leftarrow}$ NSs $5^{\prime}$

mRNA

Transcription

Antigenome

$5^{\prime}$

Replication

Genome

Transcription

mRNA
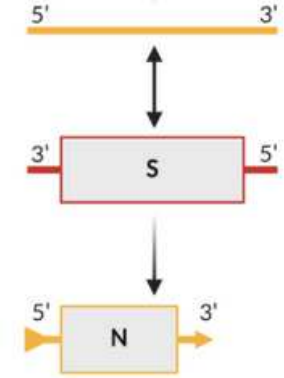

$\mathrm{S} / \mathrm{cS}$
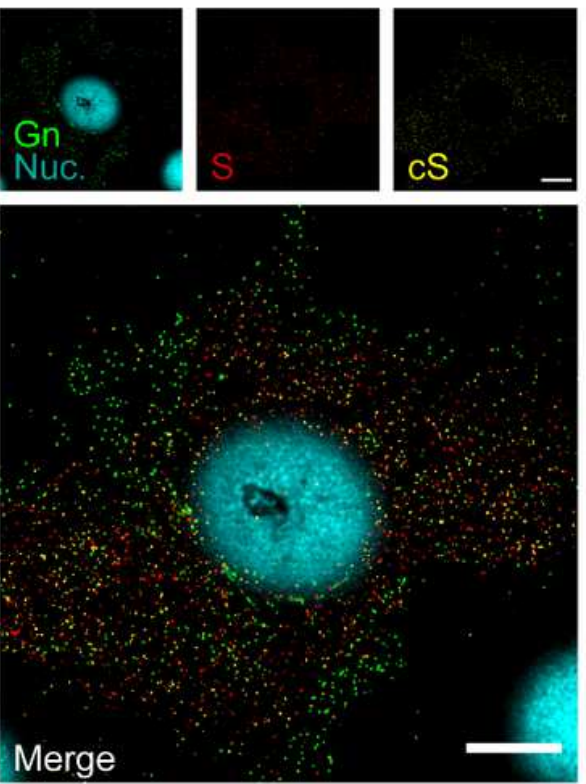

C

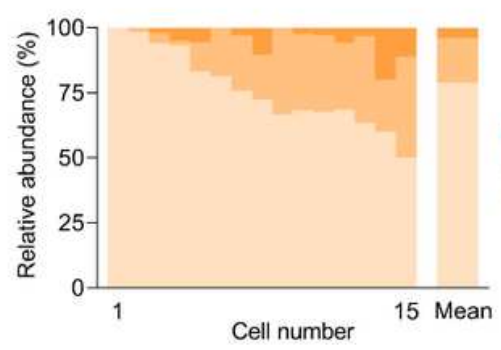

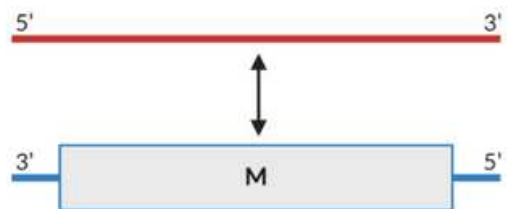

5

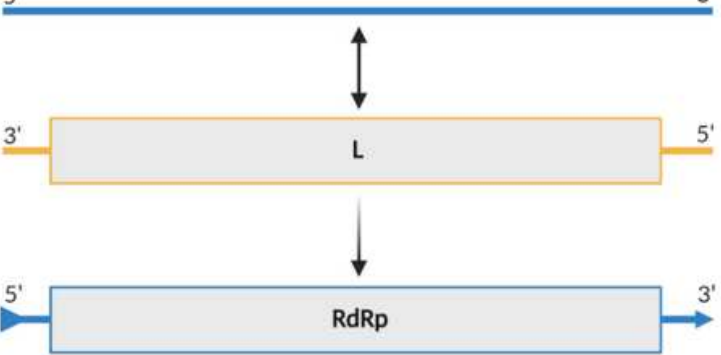

$\mathrm{M} / \mathrm{CM}$
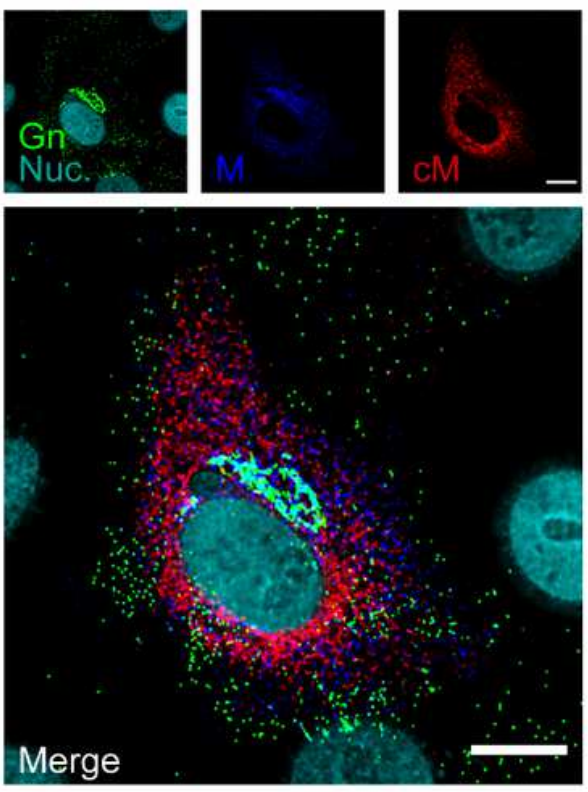

$\mathrm{M} / \mathrm{cM}$ ratio $\approx 9: 1$

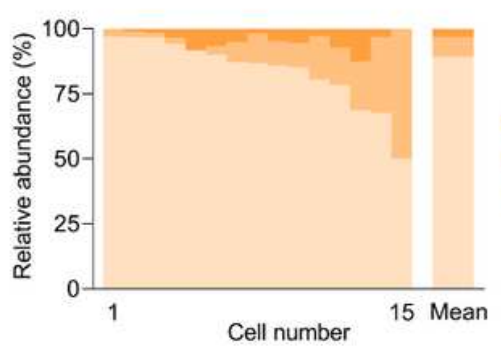

$\mathrm{L} / \mathrm{CL}$
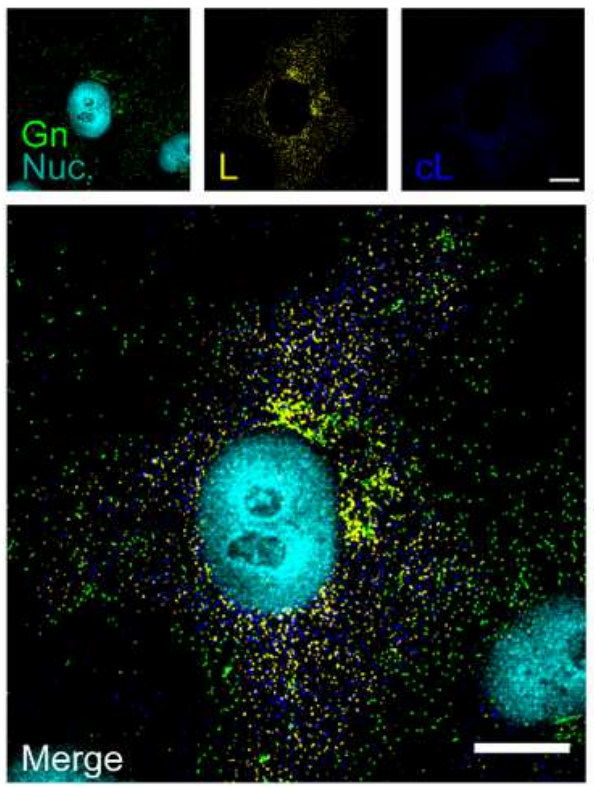

$\mathrm{L} / \mathrm{CL}$ ratio $\approx 14: 1$

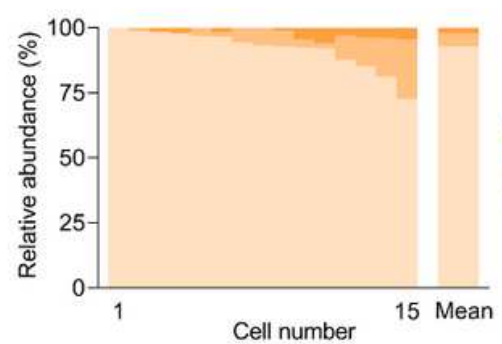

\section{Figure 6}

Single-molecule RNA FISH-immunofluorescence on vRNA-cRNA pairs of RVFV infected mammalian cells. a Schematic representation of the replication and transcription of RVFV genome segments. Here, we refer to viral genome replication intermediates (antigenomes) and mRNA transcripts as cRNAs. RVFV S segment uses an ambisense coding strategy to generate mRNAs from both the genomic-sense and antigenomic-sense RNAs. b Visualization of vRNPs, cRNAs and progeny virions of RVFV infected cells. Vero E6 cells were infected with RVFV (MOI 0.75-1.00) and cells were fixed at 8-10 h post-infection. 
Samples were hybridized against paired targets (i.e. S-cS segments, M-cM segments and L-cL segments). The $S$ segment ( $N$ gene; red), M segment (polyprotein gene; blue) and $L$ segment (RdRp gene; yellow) were hybridized using probe sets labelled with CAL Fluor Red 610, Quasar 670 and Quasar 570, respectively. The $\mathrm{cS}$ segment ( $\mathrm{N}$ gene; yellow), cM segment (polyprotein gene; red) and $\mathrm{cL}$ segment (RdRp gene; blue) were hybridized using probe sets labelled with Quasar 570, CAL Fluor Red 610 and Quasar 670 , respectively. Progeny RVFV particles (green) were detected with antibody 4-D448 targeting the Gn glycoprotein in combination with Alexa Fluor 488-conjugated secondary antibodies. Cell nuclei (cyan) were visualized with DAPI. Individual spots, each representing either a vRNP, a cRNA or a virus particle were detected counted and assessed for co-localization in ImageJ with the plugin ComDet. Main images are merged maximum intensity projections of four channels (individual channels shown on top). Due to a higher fluorescence intensity of the green channel compared to the other channels, spots co-localizing with the glycoprotein may sometimes appear masked and not entirely evident in merged images. Scale bars, $10 \mu \mathrm{m}$. c Quantification of the S, M, L vRNPs and their corresponding cRNAs in RVFV progeny virions. Genome compositions of the virions are expressed astheir abundance relative to the amount of virions in which at least one vRNP or cRNA was detected. Graphs show the composition results of virions released by single cells ( $n=15$ cells per combination; more than 3,900 virions per combination) and means. vRNA/cRNA ratios are indicated. $\mathrm{cS}$, complementary $\mathrm{S}$ segment; $\mathrm{CM}$, complementary $\mathrm{M}$ segment; $\mathrm{CL}$, complementary $\mathrm{L}$ segment.

\section{Supplementary Files}

This is a list of supplementary files associated with this preprint. Click to download.

- SupplementaryMovie1.mp4

- SupplementaryMovie2.mp4

- SupplementaryMovie3.mp4

- SupplementaryMovie4.mp4

- Supplementarylnformation.pdf

- SupplementaryTable1.xlsx

- rs.pdf 